\title{
Multilinear fractional integral with rough kernel on variable exponent Morrey-Herz spaces
}

\author{
Afif Abdalmonem ${ }^{1, *}$, Omer Abdalrhman ${ }^{2}$ and Shuangping Tao ${ }^{3}$ \\ College of Science, Dalanj University, Sudan. \\ College of Education, Shendi University, Sudan.; humoora@gmail.com \\ 3 College of Mathematics and Statistics, Northwest Normal University, China.; taosp@nwnu.edu.cn \\ * Correspondence: afeefy86@gmail.com
}

Received: 25 February 2019; Accepted: 24 May 2019; Published: 30 May 2019.

\begin{abstract}
In this article, we study a class of the multilinear fractional integral with rough kernel on Morrey-Herz space with $p(\cdot), q(\cdot), \alpha(\cdot)$. By using the properties of the variable exponent spaces, the boundedness of the multilinear fractional integral operator is obtained on variable nonhomogeneous Morrey-Herz spaces $M K_{q(\cdot), p(\cdot)}^{\alpha(\cdot), \lambda}\left(\mathbb{R}^{n}\right)$.
\end{abstract}

Keywords: Multilinear fractional integral, rough kernel, BMO function, Lipschitz function, Morrey-Herz spaces with variable exponents.

MSC: 2010: 42B20, 42B25.

\section{Introduction}

$\mathbf{S}$ uppose that $S^{n-1}(n>1)$ denote the unit sphere in $\mathbb{R}^{n}$ with the normalized Lebesgue measure $\mathrm{d} \sigma\left(x^{\prime}\right)$. Let $\Omega \in L^{s}\left(S^{n-1}\right)(1<s<\infty)$ be a homogeneous function of degree zero on $\mathbb{R}^{n}$. The multilinear fractional integral operator with rough kernel $T_{\Omega, \mu}^{A}(0<\mu<n)$ is defined by

$$
T_{\Omega, \mu}^{A} f(x)=\int_{\mathbb{R}^{n}} \frac{\Omega(x-y)}{|x-y|^{n-\mu+m}} R_{m+1}(A ; x, y) f(y) d y,
$$

where $A$ is a function defined on $\mathbb{R}^{n}$ and $R_{m+1}(A ; x, y)$ is the $m$ th reminder of Taylor series of $A$ at $x$ about $y$. More precisely

$$
R_{m+1}(A ; x, y)=A(x)-\sum_{|\gamma| \leq m} \frac{1}{\gamma !} D^{\gamma} A(y)(x-y)^{\gamma},
$$

when $m=1, T_{\Omega, \mu}^{A}$ is just the commutators of the fractional integral with rough kernel with function $A$.

$$
T_{\Omega, \mu}^{A} f(x)=\int_{\mathbb{R}^{n}} \frac{\Omega(x-y)}{|x-y|^{n-\mu}}(A(x)-A(y)) f(y) d y .
$$

The multilinear fractional maximal operator with rough kernel is defined as

$$
M_{\Omega, \mu}^{A} f(x)=\sup _{r>0} \frac{1}{r^{(n-\mu+m)}} \int_{|x-y|<r}\left|\Omega(x-y) R_{m+1}(A ; x, y) f(y)\right| d y .
$$

In 1975, Coifman and Meyer [1] introduced multilinear integral and the boundedness of the multilinear fractional integral operator on Lebesgue spaces established in [2-4]. Li and Tao [5] discussed the boundedness of multilinear commutators with rough kernels on Morrey-Herz spaces.

On the other hand, the theory of the variable exponent function spaces has been rapidly developed after it was introduced by Kováčik and Rákosník [6]. After that, many researchers work in this direction has been done, see for example [7-13]. The boundedness of the multilinear fractional integral operator on variable Lebesgue spaces are established in [14,15]. Recently, $\mathrm{Lu}$ and $\mathrm{Zhu}$ [16] established the boundedness of the multilinear Calderón-Zygmund singular operators on Morrey-Herz spaces with variable exponents. 
In this article, we study the boundedness of the multilinear fractional integral operator and multilinear fractional maximal operator with rough kernels on variable exponent Lebesgue spaces. The boundedness of the multilinear fractional integral operator is established on Morrey-Herz spaces $M K_{q(\cdot), p(\cdot)}^{\alpha(\cdot), \lambda}\left(\mathbb{R}^{n}\right)$.

Throughout this paper, let $|E|$ be a Lebesgue measurable set in $\mathbb{R}^{n}$ with measure $|E|>0$ and $\chi_{E}$ be its characteristic function. We shall recall some definitions.

Definition 1. [7]. Let $p(\cdot): E \rightarrow[1, \infty)$ be a measurable function, the variable exponent Lebesgue spaces $L^{p(\cdot)}(E)$ is defined as

$$
L^{p(\cdot)}(E)=\left\{f \text { is measurable }: \int_{E}\left(\frac{|f(x)|}{\eta}\right)^{p(x)} d x<\infty \text { for some constant } \eta>0\right\} .
$$

The space $L_{l o c}^{p(\cdot)}(E)$ is defined as

$$
L_{\text {loc }}^{p(\cdot)}(E)=\left\{\mathrm{f} \text { is measurable }: f \in L^{p(\cdot)}(K) \text { for all compact } K \subset E\right\} .
$$

The relation between Lebesgue spaces $L^{p(\cdot)}(E)$ and Banach spaces is defined as

$$
\|f\|_{L^{p(\cdot)}(E)}=\inf \left\{\eta>0: \int_{E}\left(\frac{|f(x)|}{\eta}\right)^{p(x)} d x \leq 1\right\} .
$$

We denote $p_{-}=\operatorname{essinf}\{p(x): x \in E\}, p_{+}=\operatorname{esssup}\{p(x): x \in E\}$. Then $\mathcal{P}(E)$ consists of all $p(\cdot)$ satisfying $p_{-}>1$ and $p_{+}<\infty$.

Next, we give the definition of Morrey-Herz space with variable exponents $q(\cdot), p(\cdot), \alpha(\cdot)$. Let $B_{k}=\{x \in$ $\left.\mathbb{R}^{n}:|x| \leq 2^{k}\right\}, C_{k}=B_{k} \backslash B_{k-1}, \chi_{k}=\chi_{C_{k}}, k \in \mathbb{Z}$.

Definition 2. [18]. Let $q(\cdot), p(\cdot) \in \mathcal{P}\left(\mathbb{R}^{n}\right), 0 \leq \lambda<\infty$ and $\alpha(\cdot): \mathbb{R}^{n} \longrightarrow \mathbb{R}$ with $\alpha \in L^{\infty}\left(\mathbb{R}^{n}\right)$. The nonhomogeneous Morrey-Herz space with variable exponents $M K_{q(\cdot), p(\cdot)}^{\alpha(\cdot), \lambda}\left(\mathbb{R}^{n}\right)$ is defined as

$$
M K_{q(\cdot), p(\cdot)}^{\alpha(\cdot), \lambda}\left(\mathbb{R}^{n}\right)=\left\{f \in L_{\text {loc }}^{p(\cdot)}\left(\mathbb{R}^{n} \backslash\{0\}\right):\|f\|_{M K_{q(\cdot), p(\cdot)}^{\alpha(\cdot), \lambda}\left(\mathbb{R}^{n}\right)}<\infty\right\},
$$

where

$$
\|f\|_{M K_{q(\cdot), p(\cdot)}^{\alpha(\cdot), \lambda}\left(\mathbb{R}^{n}\right)}=\inf \left\{\eta>0: \sup _{k_{0} \in z} 2^{-k_{0} \lambda} \sum_{k=0}^{k_{0}}\left\|\left(\frac{2^{k \alpha(\cdot)}\left|f \chi_{k}\right|}{\eta}\right)^{q(\cdot)}\right\|_{L^{p(\cdot)}} \leq 1\right\} .
$$

The homogeneous Morrey-Herz space with variable exponents $M \dot{K}_{q(\cdot), p(\cdot)}^{\alpha(\cdot), \lambda}\left(\mathbb{R}^{n}\right)$ is defined as

$$
M \dot{K}_{q(\cdot), p(\cdot)}^{\alpha(\cdot), \lambda}\left(\mathbb{R}^{n}\right)=\left\{f \in L_{\text {loc }}^{p(\cdot)}\left(\mathbb{R}^{n} \backslash\{0\}\right):\|f\|_{M \dot{K}_{q(\cdot), p(\cdot)}^{\alpha(\cdot), \lambda}\left(\mathbb{R}^{n}\right)}<\infty\right\},
$$

where

$$
\|f\|_{M \dot{K}_{q(\cdot), p(\cdot)}^{\alpha(\cdot), \lambda}\left(\mathbb{R}^{n}\right)}=\inf \left\{\eta>0: \sup _{k_{0} \in z} 2^{-k_{0} \lambda} \sum_{k=-\infty}^{k_{0}}\left\|\left(\frac{2^{k \alpha(\cdot)}\left|f \chi_{k}\right|}{\eta}\right)^{q(\cdot)}\right\|_{L^{p(\cdot)}} \leq 1\right\} .
$$

\section{Preliminaries}

In this section, we give some properties of variable exponents that will be helpful in proving our main results.

Proposition 1. [7]. If $p(\cdot) \in \mathcal{P}\left(\mathbb{R}^{n}\right)$ satisfies the follows inequalities:

$$
\begin{aligned}
& |p(x)-p(y)| \leq \frac{-C}{\log (|x-y|)},|x-y| \leq 1 / 2 ; \\
& |p(x)-p(y)| \leq \frac{C}{\log (e+|x|)},|y| \geq|x| .
\end{aligned}
$$


then, we have $p(\cdot) \in \mathfrak{B}\left(\mathbb{R}^{n}\right)$.

Proposition 2. [14]. Suppose that $p_{1}(\cdot) \in \mathfrak{B}\left(\mathbb{R}^{n}\right), \Omega \in L^{r}\left(S^{n-1}\right), 0<\mu \leq \frac{n}{\left(p_{1}\right)_{+}}, \frac{1}{p_{1}(x)}-\frac{1}{p_{2}(x)}=\frac{\mu}{n}$, then for all $f \in L^{p_{1}(\cdot)}\left(\mathbb{R}^{n}\right)$, we have

$$
\left\|M_{\Omega, \mu} f\right\|_{L^{p_{2}(\cdot)}\left(\mathbb{R}^{n}\right)} \leq C\|f\|_{L^{p_{1}(\cdot)}\left(\mathbb{R}^{n}\right)} \cdot
$$

Now, we recall some lemmas.

Lemma 1. [7].

1. Let $p(\cdot): \mathbb{R}^{n} \rightarrow[1, \infty)$, for all function $f$ and $g$, then

$$
\int_{\mathbb{R}^{n}}|f(x) g(x)| \mathrm{d} x \leq C\|f\|_{L^{p(\cdot)\left(\mathbb{R}^{n}\right)}}\|g\|_{L^{p^{\prime}(\cdot)\left(\mathbb{R}^{n}\right)}} .
$$

2. If $p(\cdot), q(\cdot), r(\cdot) \in \mathbb{R}^{n}, p(\cdot)$ and $\frac{1}{p(\cdot)}=\frac{1}{q(\cdot)}+\frac{1}{r(\cdot)}$. Then there exists a constant $C$ such that for all $f \in L^{q(\cdot)}\left(\mathbb{R}^{n}\right), g \in L^{r(\cdot)}\left(\mathbb{R}^{n}\right)$, we have

$$
\|f g\|_{L^{p(\cdot)}} \leq C\|f\|_{L^{g(\cdot)}\left(\mathbb{R}^{n}\right)}\|g\|_{L^{r(\cdot)}\left(\mathbb{R}^{n}\right)} .
$$

Lemma 2. [7]. Let $x \in \mathbb{R}^{n}$ and $\frac{1}{p(\cdot)}=\frac{1}{q}+\frac{1}{\tilde{q}(\cdot)}$, then for all measurable function $f$ and $g$, we have

$$
\|f(x) g(x)\|_{L^{p(\cdot)}\left(\mathbb{R}^{n}\right)} \leq C\|g(x)\|_{L^{q}\left(\mathbb{R}^{n}\right)}\|f(x)\|_{L^{\tilde{q}(\cdot)}\left(\mathbb{R}^{n}\right)} .
$$

Lemma 3. [9]. Suppose that $p(\cdot) \in \mathfrak{B}\left(\mathbb{R}^{n}\right)$ and $0<p^{-} \leq p^{+}<\infty$, then we have

1. for any cube $|Q| \leq 2^{n}$, and all the $\chi \in Q$, we have $\left\|\chi_{Q}\right\|_{L^{p(\cdot)}} \approx|Q|^{1 / p(x)}$,

2. for any cube $|Q| \geq 1$, we have $\left\|\chi_{Q}\right\|_{L^{p(\cdot)}} \approx|Q|^{1 / p_{\infty}}$, where $p_{\infty}=\lim _{x \rightarrow \infty} p(x)$.

Lemma 4. [11]. If $p(\cdot) \in \mathfrak{B}\left(\mathbb{R}^{n}\right)$, then there exist constants $\delta_{1}, \delta_{2}, C>0$ such that for all balls $B$ in $\mathbb{R}^{n}$ and all measurable subset $S \subset B$, we have

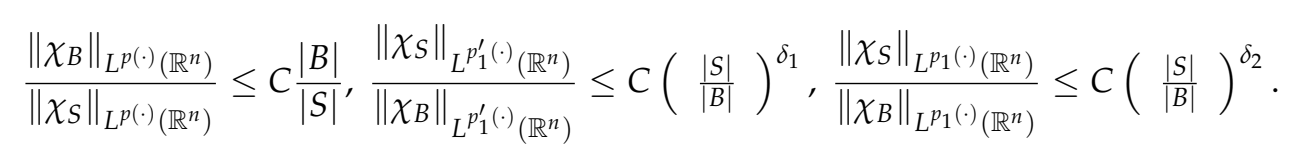

Lemma 5. [19]. For $p(\cdot) \in \mathfrak{B}\left(\mathbb{R}^{n}\right)$, there exists constant $C>0$ such that for any balls $B$ in $\mathbb{R}^{n}$, we have

$$
\frac{1}{|B|}\left\|\chi_{B}\right\|_{L^{p(\cdot)}\left(\mathbb{R}^{n}\right)}\left\|\chi_{B}\right\|_{L^{p^{\prime}(\cdot)\left(\mathbb{R}^{n}\right)}} \leq C
$$

Lemma 6. [13]. Let $p(\cdot), q(\cdot) \in \mathcal{P}\left(\mathbb{R}^{n}\right)$. If $f \in L^{p(\cdot) q(\cdot)}$, then

$$
\min \left(\|f\|_{L^{p(\cdot) q(\cdot)}}^{q_{+}},\|f\|_{L^{p(\cdot) q(\cdot)}}^{q_{-}}\right) \leq\left\|\left.|f|\right|^{q(\cdot)}\right\|_{L^{p(\cdot)}} \leq \max \left(\|f\|_{L^{p(\cdot) q(\cdot)}}^{q_{+}},\|f\|_{L^{p(\cdot) q(\cdot)}}^{q_{-}}\right) .
$$

Lemma 7. [20]. Let $b \in B M O\left(\mathbb{R}^{n}\right)$, where $n$ is a positive integer, and let the constant $C>0$. Then for any $l, j \in \mathbb{Z}$ with $l>j$, we have

1. $C^{-1}\|b\|_{*}^{n} \leq \sup _{B} \frac{1}{\left\|\chi_{B}\right\|_{L^{p(\cdot)}\left(\mathbb{R}^{n}\right)}}\left\|\left(b-b_{B}\right)^{n} \chi_{B}\right\|_{L^{p(\cdot)}\left(\mathbb{R}^{n}\right)} \leq C\|b\|_{*}^{n}$

2. $\left\|\left(b-b_{B_{j}}\right)^{n} \chi_{B_{k}}\right\|_{L^{p(\cdot)}\left(\mathbb{R}^{n}\right)} \leq C(l-j)^{n}\|b\|_{*}^{n}\left\|\chi_{B_{k}}\right\|_{L^{p(\cdot)}\left(\mathbb{R}^{n}\right)}$.

Lemma 8. [2]. For any $\varepsilon>0$ with $0<\mu-\varepsilon<\mu+\varepsilon<n$, we have

$$
\left|T_{\Omega, \mu}^{A} f(x)\right| \leq C\left[M_{\Omega, \mu+\varepsilon}^{A} f(x)\right]^{\frac{1}{2}}\left[M_{\Omega, \mu-\varepsilon}^{A} f(x)\right]^{\frac{1}{2}} .
$$

Lemma 9. [4]. Let $A$ be a function with derivatives of order $m$ in $\dot{\Lambda}_{\beta}(0<\beta<1)$. Then there exists a constant $C>0$ such that

$$
\left|R_{m+1}(A ; x, y)\right| \leq C\left(\sum_{|\gamma|=m}\left\|D^{\gamma} A\right\|_{\lambda_{\beta}}\right)|x-y|^{m+\beta} .
$$


Lemma 10. [21]. Let $b(x)$ be a function on $\mathbb{R}^{n}$ and $D^{\gamma} b \in L_{l o c}^{q}\left(\mathbb{R}^{n}\right)$, where $q>n$, then

$$
\left|R_{m}(b, x, y)\right| \leq C|x-y|^{m} \sum_{|\gamma|=m}\left(\frac{1}{B(x, y)} \int_{B(x, y)}\left|D^{\gamma} b(z)\right| d z\right)^{\frac{1}{q}} .
$$

where $B(x, y)$ is the cube centered at $x$ and having diameter $5 \sqrt{n}|x-y|$.

Lemma 11. [7]. Let $q(\cdot) \in \mathcal{P}\left(\mathbb{R}^{n}\right)$ and $q^{+}<\infty$, then for any $s>0$, we have

$$
\left\||f|^{s}\right\|_{q(\cdot)\left(\mathbb{R}^{n}\right)}=\|f\|_{s q(\cdot)\left(\mathbb{R}^{n}\right)}^{s}
$$

Lemma 12. Let $D^{\gamma} A \in B M O\left(\mathbb{R}^{n}\right)(|\gamma|=|m|, m \geq 1), \Omega \in L^{r}\left(S^{n-1}\right), p(\cdot) \in \mathfrak{B}\left(\mathbb{R}^{n}\right)$ and $\frac{1}{p_{1}(x)}-\frac{1}{p_{2}(x)}=\frac{\mu}{n}$, then we have

$$
\left\|M_{\Omega, \mu}^{A} f(x)\right\|_{L^{p_{2}(\cdot)}\left(\mathbb{R}^{n}\right)} \leq C \sum_{|\gamma|=m}\left\|D^{\gamma} A\right\|_{B M O\left(\mathbb{R}^{n}\right)}\|f\|_{L^{p_{1}(\cdot)}\left(\mathbb{R}^{n}\right)^{\prime}}
$$

where $C$ is independent of $f$ and $A$.

Proof. If $\widetilde{M}_{\Omega, \mu}^{A}$ is defined by:

$$
\widetilde{M}_{\Omega, \mu}^{A} f(x)=\sup _{r>0} \frac{1}{r^{(n-\mu+m)}} \int_{\frac{r}{2}<|x-y|<r}\left|\Omega(x-y) R_{m+1}(A ; x, y) f(y)\right| \mathrm{d} y .
$$

Let $Q(x, r)$ be the cube centered at $x$ and having diameter $5 \sqrt{n r}$. If $\frac{r}{2}<|x-y|<r$, by Lemma 10 , we have

$$
\begin{aligned}
\left|R_{m+1}(A ; x, y)\right|= & \left|R_{m+1}\left(A_{k} ; x, y\right)\right| \leq\left|R_{m}\left(A_{k} ; x, y\right)\right|+\sum_{|\gamma|=m} \frac{1}{\gamma !}\left|D^{\gamma} A_{k}(y)\right||x-y|^{m} \\
& \leq C|x-y|^{m}\left(\sum_{|\gamma|=m}\left\|D^{\gamma} A\right\|_{B M O\left(\mathbb{R}^{n}\right)}+\sum_{|\gamma|=m}\left|D^{\gamma} A(y)-m_{B_{k}}\left(D^{\gamma} A\right)\right|\right) .
\end{aligned}
$$

By using Hölder's inequality, we get

$$
\begin{aligned}
\widetilde{M}_{\Omega, \mu}^{A} f(x)= & \sup _{r>0} \frac{1}{r^{n-\mu}} \int_{\frac{r}{2}<|x-y|<r}\left(\sum_{|\gamma|=m}\left\|D^{\gamma} A\right\|_{B M O\left(\mathbb{R}^{n}\right)}+\sum_{|\gamma|=m}\left|D^{\gamma} A(y)-m_{B_{k}}\left(D^{\gamma} A\right)\right|\right) \\
& \times|\Omega(x-y) f(y)| \text { dy } \\
\leq & C \sum_{|\gamma|=m}\left\|D^{\gamma} A\right\|_{B M O\left(\mathbb{R}^{n}\right)}\left(M_{|\Omega|^{t}, \mu t}\left(|f|^{t}\right)(x)\right)^{\frac{1}{t}} .
\end{aligned}
$$

By the boundedness of the fractional maximal operator on $L^{p(\cdot)}\left(\mathbb{R}^{n}\right)$ spaces, we obtain that

$$
\begin{aligned}
\left\|\widetilde{M}_{\Omega, \mu}^{A} f(x)\right\|_{L^{p_{2}(\cdot)\left(\mathbb{R}^{n}\right)}} & \leq C \sum_{|\gamma|=m}\left\|D^{\gamma} A\right\|_{B M O\left(\mathbb{R}^{n}\right)}\left\|M_{|\Omega|^{t}, \mu t}\left(|f|^{t}\right)(x)\right\|_{L^{\frac{1}{t}}}^{\frac{1}{t}(\cdot)} \\
& \leq C \sum_{|\gamma|=m}\left\|D^{\gamma} A\right\|_{B M O\left(\mathbb{R}^{n}\right)}\left\||f|^{t}\right\|_{L^{\frac{1}{t}}}^{\frac{p_{1}(\cdot)}{t}} \\
& \leq C \sum_{|\gamma|=m}\left\|D^{\gamma} A\right\|_{B M O\left(\mathbb{R}^{n}\right)}\|f\|_{L^{p_{1}(\cdot)}\left(\mathbb{R}^{n}\right)^{\prime}}
\end{aligned}
$$

Since $M_{\Omega, \mu}^{A} f(x) \leq \widetilde{M}_{\Omega, \mu}^{A} f(x)$ for all $x \in \mathbb{R}^{n}$, we have

$$
\begin{aligned}
\left\|M_{\Omega, \mu}^{A} f(x)\right\|_{L^{p_{2}(\cdot)\left(\mathbb{R}^{n}\right)}} & \leq\left\|\widetilde{M}_{\Omega, \mu}^{A} f(x)\right\|_{L^{p_{2}(\cdot)}\left(\mathbb{R}^{n}\right)} \\
& \leq C \sum_{|\gamma|=m}\left\|D^{\gamma} A\right\|_{B M O\left(\mathbb{R}^{n}\right)}\|f\|_{L^{p_{1}(\cdot)}\left(\mathbb{R}^{n}\right)} .
\end{aligned}
$$


Then, we get

$$
\left\|M_{\Omega, \mu}^{A} f(x)\right\|_{L^{p_{2}(\cdot)\left(\mathbb{R}^{n}\right)}} \leq C \sum_{|\gamma|=m}\left\|D^{\gamma} A\right\|_{B M O\left(\mathbb{R}^{n}\right)}\|f\|_{L^{p_{1}(\cdot)}\left(\mathbb{R}^{n}\right)^{\prime}} .
$$

This completes the proof of Lemma 12.

Lemma 13. Let $D^{\gamma} A \in B M O\left(\mathbb{R}^{n}\right)(|\gamma|=|m|, m \geq 1), \Omega \in L^{r}\left(S^{n-1}\right), p(\cdot) \in \mathfrak{B}\left(\mathbb{R}^{n}\right)$ and $\frac{1}{p_{1}(x)}-\frac{1}{p_{2}(x)}=\frac{\mu}{n}$, then we have

$$
\left\|T_{\Omega, \mu}^{A} f(x)\right\|_{L^{p_{2}(\cdot)\left(\mathbb{R}^{n}\right)}} \leq C \sum_{|\gamma|=m}\left\|D^{\gamma} A\right\|_{B M O\left(\mathbb{R}^{n}\right)}\|f\|_{L^{p_{1}(\cdot)\left(\mathbb{R}^{n}\right)}} .
$$

where $C$ is independent of $f$ and $A$.

Proof. Let $0<\varepsilon<\min (\mu, n-\mu)$, and $r(\cdot): \mathbb{R}^{n} \longrightarrow[1,+\infty)$, and let

$$
\begin{aligned}
& \frac{1}{p_{1}(\cdot)}-\frac{1}{\frac{r(\cdot) p_{2}(\cdot)}{2}}=\frac{\mu-\varepsilon}{2}, \\
& \frac{1}{p_{1}(\cdot)}-\frac{1}{\frac{r^{\prime}(\cdot) p_{2}(\cdot)}{2}}=\frac{\mu+\varepsilon}{2} .
\end{aligned}
$$

By Lemma 8 and applying Hölder's inequality, we have

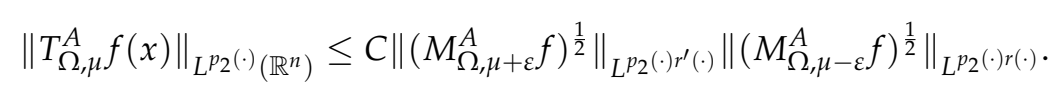

Since

$$
\begin{aligned}
\left\|\left(M_{\Omega, \mu-\varepsilon}^{A} f\right)^{\frac{1}{2}}\right\|_{L^{p_{2}(\cdot) r(\cdot)}} & \leq C\left\|\left(M_{\Omega, \mu-\varepsilon}^{A} f\right)\right\|_{L^{\frac{p_{2}(\cdot) r(\cdot)}{2}}}^{\frac{1}{2}} \\
& \leq C\left(\sum_{|\gamma|=m}\left\|D^{\gamma} A\right\|_{B M O\left(\mathbb{R}^{n}\right)}\right)^{\frac{1}{2}}\|f\|_{L^{p_{1}(\cdot)}\left(\mathbb{R}^{n}\right)}^{\frac{1}{2}} .
\end{aligned}
$$

Similar way, we concluded that

$$
\left\|\left(M_{\Omega, \mu+\varepsilon}^{A} f\right)^{\frac{1}{2}}\right\|_{L^{p_{2}(\cdot) r^{\prime}(\cdot)}} \leq C\left(\sum_{|\gamma|=m}\left\|D^{\gamma} A\right\|_{B M O\left(\mathbb{R}^{n}\right)}\right)^{\frac{1}{2}}\|f\|_{L^{p_{1}(\cdot)}\left(\mathbb{R}^{n}\right)^{\prime}}^{\frac{1}{2}}
$$

then, we have

$$
\left\|T_{\Omega, \mu}^{A} f(x)\right\|_{L^{p_{2}(\cdot)}\left(\mathbb{R}^{n}\right)} \leq C \sum_{|\gamma|=m}\left\|D^{\gamma} A\right\|_{B M O\left(\mathbb{R}^{n}\right)}\|f\|_{L^{p_{1}(\cdot)}\left(\mathbb{R}^{n}\right)} .
$$

This completes the proof of Lemma 13.

\section{Main results}

In this section, we investigate the boundedness of the multilinear fractional integral operator with rough kernel on variable nonhomogeneous Morrey-Herz spaces $M K_{q(\cdot), p(\cdot)}^{\alpha(\cdot),}\left(\mathbb{R}^{n}\right)$.

Theorem 1. Suppose that $D^{\gamma} A \in B M O\left(\mathbb{R}^{n}\right)(|\gamma|=|m|, m \geq 1)$. Let $0<\mu<n, \Omega \in L^{r}\left(s^{n-1}\right), q_{1}(\cdot), q_{2}(\cdot) \in$ $\mathcal{P}\left(\mathbb{R}^{n}\right)$ with $\left(q_{2}\right)_{-} \geq\left(q_{1}\right)_{+}$, and $p_{1}(\cdot), p_{2}(\cdot) \in \mathfrak{B}\left(\mathbb{R}^{n}\right)$ satisfy $0<\mu \leq \frac{n}{\left(p_{1}\right)_{+}}, \frac{1}{p_{1}(x)}-\frac{1}{p_{2}(x)}=\frac{\mu}{n}$. If $\left(\lambda_{1}\right)\left(q_{2}\right)_{+}=$ $\left(\lambda_{2}\right)\left(q_{1}\right)_{-}$and $\mu-n \delta_{2}+\left(\lambda_{1}\right) /\left(q_{1}\right)_{-}<\alpha_{+}<n \delta_{1}+\frac{n}{r}+\left(\lambda_{1}\right) /\left(q_{1}\right)_{-}$. Then $T_{\Omega, \mu}^{A}$ is bounded from $M K_{q_{1}(\cdot), p_{1}(\cdot)}^{\alpha_{+}, \lambda_{1}}\left(\mathbb{R}^{n}\right)$ to $M K_{q_{2}(\cdot), p_{2}(\cdot)}^{\alpha(\cdot), \lambda_{2}}\left(\mathbb{R}^{n}\right)$.

Proof. Let $D^{\gamma} A \in B M O\left(\mathbb{R}^{n}\right), f \in M K_{q_{1}(\cdot), p_{1}(\cdot)}^{\alpha_{+}, \lambda_{1}}\left(\mathbb{R}^{n}\right)$, we write

$$
f(x)=\sum_{j=0}^{\infty} f(x) \chi_{j}(x)=\sum_{j=0}^{\infty} f_{j}(x)
$$


Open J. Math. Sci. 2019, 3, 167-183

172

By the definition of $M K_{q(\cdot), p(\cdot)}^{\alpha(\cdot),}\left(\mathbb{R}^{n}\right)$, we have

$$
\left\|T_{\Omega, \mu}^{A}(f) \chi_{k}\right\|_{M K_{q_{2}(\cdot), p_{2}(\cdot)}^{\alpha(\cdot), \lambda_{2}}\left(\mathbb{R}^{n}\right)}=\inf \left\{\eta>0: \sup _{k_{0} \in z} 2^{-k_{0} \lambda_{2}} \sum_{k=0}^{k_{0}}\left\|\left(\frac{2^{k \alpha(\cdot)}\left|T_{\Omega, \mu}^{A}(f) \chi_{k}\right|}{\eta}\right)^{q_{2}(\cdot)}\right\|_{L_{L_{2}}^{p_{2}(\cdot)}} \leq 1\right\} .
$$

For any $k_{0} \in z$, we see that

$$
\begin{aligned}
& 2^{-k_{0} \lambda_{2}} \sum_{k=0}^{k_{0}}\left\|\left(\frac{2^{k \alpha(\cdot)}\left|T_{\Omega, \mu}^{A}(f) \chi_{k}\right|}{\eta}\right)^{q_{2}(\cdot)}\right\|_{L_{\frac{2}{q_{2}(\cdot)}}} \leq 2^{-k_{0} \lambda_{2}} \sum_{k=0}^{k_{0}}\left\|\left(\frac{2^{k \alpha(\cdot)}\left|\sum_{j=0}^{\infty} T_{\Omega, \mu}^{A}\left(f_{j}\right) \chi_{k}\right|}{\eta_{11}+\eta_{12}+\eta_{13}}\right)^{q_{2}(\cdot)}\right\|_{L_{\frac{p_{2}(\cdot)}{q_{2}(\cdot)}}} \\
& \leq 2^{-k_{0} \lambda_{2}} \sum_{k=0}^{k_{0}}\left\|\left(\frac{2^{k \alpha(\cdot)}\left|\sum_{j=0}^{k-2} T_{\Omega, \mu}^{A}\left(f_{j}\right) \chi_{k}\right|}{\eta_{11}}\right)^{q_{2}(\cdot)}\right\|\left\|_{L^{p_{2}(\cdot)}}+2^{-k_{0} \lambda_{2}} \sum_{k=0}^{k_{0}}\right\|\left(\frac{2^{k \alpha(\cdot)}\left|\sum_{j=k-1}^{k+1} T_{\Omega, \mu}^{A}\left(f_{j}\right) \chi_{k}\right|}{\eta_{12}}\right)^{q_{2}(\cdot)} \|_{L_{2_{2}(\cdot)}} \\
& +2^{-k_{0} \lambda_{2}} \sum_{k=0}^{k_{0}}\left\|\left(\frac{2^{k \alpha(\cdot)}\left|\sum_{j=k+2}^{\infty} T_{\Omega, \mu}^{A}\left(f_{j}\right) \chi_{k}\right|}{\eta_{13}}\right)^{q_{2}(\cdot)}\right\|_{L_{\frac{p_{2}(\cdot)}{q_{2}(\cdot)}}},
\end{aligned}
$$

where

$$
\begin{aligned}
& \eta_{11}=\left\|\sum_{j=0}^{k-2} T_{\Omega, \mu}^{A}\left(f_{j}\right) \chi_{k}\right\|_{M K_{q_{2}(\cdot), p_{2}(\cdot)}^{\alpha(\cdot) \lambda_{2}}\left(\mathbb{R}^{n}\right)} \\
& =\inf \left\{\eta>0: \sup _{k_{0} \in z} 2^{-k_{0} \lambda_{2}} \sum_{k=0}^{k_{0}}\left\|\left(\frac{2^{k \alpha(\cdot)}\left|\sum_{j=0}^{k-2} T_{\Omega, \mu}^{A}\left(f_{j}\right) \chi_{k}\right|}{\eta}\right)^{q_{2}(\cdot)}\right\|_{\frac{p_{2}(\cdot)}{q_{2}(\cdot)}} \leq 1\right\} \text {, } \\
& \eta_{12}=\left\|\sum_{k-1}^{k+1} T_{\Omega, \mu}^{A}\left(f_{j}\right) \chi_{k}\right\|_{M K_{q_{2}(\cdot), p_{2}(\cdot)}^{(\cdot \cdot), \lambda_{2}}}\left(\mathbb{R}^{n}\right) \\
& =\inf \left\{\eta>0: \sup _{k_{0} \in z} 2^{-k_{0} \lambda_{2}} \sum_{k=0}^{k_{0}}\left\|\left(\frac{2^{k \alpha(\cdot)}\left|\sum_{j=k-1}^{k+1} T_{\Omega, \mu}^{A}\left(f_{j}\right) \chi_{k}\right|}{\eta}\right)^{q_{2}(\cdot)}\right\|_{L_{\frac{p_{2}(\cdot)}{q_{2}(\cdot)}}} \leq 1\right\} \text {, } \\
& \eta_{13}=\left\|\sum_{j=k+2}^{\infty} T_{\Omega, \mu}^{A}\left(f_{j}\right) \chi_{k}\right\|_{M K_{q_{2}(\cdot), p_{2}(\cdot)}^{\alpha(\cdot), \lambda_{2}}}\left(\mathbb{R}^{n}\right) \\
& =\inf \left\{\eta>0: \sup _{k_{0} \in z} 2^{-k_{0} \lambda_{2}} \sum_{k=0}^{k_{0}}\left\|\left(\frac{2^{k \alpha(\cdot)}\left|\sum_{j=k+2}^{\infty} T_{\Omega, \mu}^{A}\left(f_{j}\right) \chi_{k}\right|}{\eta}\right)^{q_{2}(\cdot)}\right\|_{{\frac{p}{q_{2}(\cdot)}}^{q_{2}(\cdot)}} \leq 1\right\} .
\end{aligned}
$$

If $\eta=\eta_{11}+\eta_{12}+\eta_{13}$, thus

$$
2^{-k_{0} \lambda_{2}} \sum_{k=0}^{k_{0}}\left\|\left(\frac{2^{k \alpha(\cdot)}\left|T_{\Omega, \mu}^{A}\left(f_{j}\right) \chi_{k}\right|}{\eta}\right)^{q_{2}(\cdot)}\right\|_{L^{\frac{p_{2}(\cdot)}{q_{2}(\cdot)}}} \leq C
$$


That is

$$
\left\|T_{\Omega, \mu}^{A}(f) \chi_{k}\right\|_{M K_{q_{2}(\cdot), p_{2}(\cdot)}^{\alpha(\cdot), \lambda_{2}}\left(\mathbb{R}^{n}\right)} \leq C \eta \leq C\left[\eta_{11}+\eta_{12}+\eta_{13}\right] .
$$

Hence, it suffices to prove

$$
\eta_{11}, \eta_{12}, \eta_{13} \leq C \sum_{|\gamma|=m}\left\|D^{\gamma} A\right\|_{B M O\left(\mathbb{R}^{n}\right)}\|f\|_{M K_{q_{1}(\cdot), p_{1}(\cdot)}^{\alpha(\cdot), \lambda_{1}}}\left(\mathbb{R}^{n}\right)^{\prime}
$$

Denote $\eta_{1}=\|f\|_{M K_{q_{1}(\cdot), p_{1}(\cdot)}^{\alpha(\cdot), \lambda_{1}}\left(\mathbb{R}^{n}\right)}$.

Now we consider $\eta_{12}$ firstly. Applying Lemma 6, noting that $T_{\Omega, \mu}^{A}$ is bounded on $L^{p(\cdot)}\left(\mathbb{R}^{n}\right)$, it follows

$$
\begin{aligned}
& 2^{-k_{0} \lambda_{2}} \sum_{k=0}^{k_{0}}\left\|\left(\frac{2^{k \alpha(\cdot)}\left|\sum_{j=k-1}^{k+1} T_{\Omega, \mu}^{A}\left(f_{j}\right) \chi_{k}\right|}{\eta_{1} \sum_{|\gamma|=m}\left\|D^{\gamma} A\right\|_{B M O\left(\mathbb{R}^{n}\right)}}\right)^{q_{2}(\cdot)}\right\|_{L^{p_{2}(\cdot)}} \\
& \leq 2^{-k_{0} \lambda_{2}} \sum_{k=0}^{k_{0}(\cdot)}\left\|\frac{2^{k \alpha(\cdot) \mid} \sum_{j=k-1}^{k+1} T_{\Omega, \mu}^{A}\left(f_{j}\right) \chi_{k} \mid}{\eta_{1} \sum_{|\gamma|=m}\left\|D^{\gamma} A\right\|_{B M O\left(\mathbb{R}^{n}\right)}}\right\|_{L^{p_{2}(\cdot)}}^{\left(q_{2}^{1}\right)_{k}} \\
& \leq 2^{-k_{0} \lambda_{2}} \sum_{k=0}^{k_{0}}\left(\sum_{j=k-1}^{k+1}\left\|\frac{2^{(k-j) \alpha_{+}} 2^{j \alpha_{+}}\left|T_{\Omega, \mu}^{A}\left(f_{j}\right) \chi_{k}\right|}{\eta_{1} \sum_{|\gamma|=m}\left\|D^{\gamma} A\right\|_{B M O\left(\mathbb{R}^{n}\right)}}\right\|_{L^{p_{2}(\cdot)}}\right)^{\left(q_{2}^{1}\right)_{k}} \\
& \leq 2^{-k_{0} \lambda_{2}} \sum_{k=0}^{k_{0}}\left(\sum_{j=k-1}^{k+1}\left\|\frac{2^{j \alpha_{+}}\left|f_{j}\right|}{\eta_{1}}\right\|_{L^{p_{1}(\cdot)}}\right)^{\left(q_{2}^{1}\right)_{k}},
\end{aligned}
$$

where

$$
\left(q_{2}^{1}\right)_{k}= \begin{cases}\left(q_{2}\right)_{-}, & \left\|\left(\frac{2^{k \alpha(\cdot) \mid} \sum_{j=k-1}^{k+1} T_{\Omega, u}^{A}\left(f_{j}\right) \chi_{k} \mid}{\eta_{1} \sum|\gamma|=m\left\|D^{\gamma} A\right\|_{B M O\left(\mathbb{R}^{n}\right)}}\right)^{q_{2}(\cdot)}\right\|_{L^{p_{2}(\cdot)}} \leq 1, \\ \left(q_{2}\right)_{+}, & \left\|\left(\frac{2^{k \alpha(\cdot) \mid} \sum_{j=k-1}^{k+1} T_{\Omega, \mu}^{A}\left(f_{j}\right) \chi_{k} \mid}{\eta_{1} \sum|\gamma|=m\left\|D^{\gamma} A\right\|_{B M O\left(\mathbb{R}^{n}\right)}}\right)^{q_{2}(\cdot)}\right\|_{L^{p_{2}(\cdot)}}>1 .\end{cases}
$$

Since $f \in M K_{q_{1}(\cdot), p_{1}(\cdot)}^{\alpha(\cdot), \lambda_{1}}\left(\mathbb{R}^{n}\right)$, we have

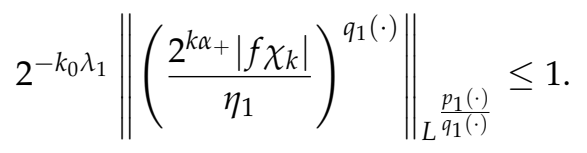

From this and again applying Lemma 6 , if $\left(q_{1}\right)_{+} \leq\left(q_{2}\right)_{-}$and $\lambda_{1}\left(q_{2}\right)_{+}=\lambda_{2}\left(q_{1}\right)_{-}$, we can obtain that

$$
\begin{aligned}
& 2^{-k_{0} \lambda_{2}} \sum_{k=0}^{k_{0}}\left\|\left(\frac{2^{k \alpha(\cdot)}\left|\sum_{j=k-1}^{k+1} T_{\Omega, \mu}^{A}\left(f_{j}\right) \chi_{k}\right|}{\eta_{1} \sum_{|\gamma|=m}\left\|D^{\gamma} A\right\|_{B M O\left(\mathbb{R}^{n}\right)}}\right)^{q_{2}(\cdot)}\right\|_{L_{\frac{p_{2}(\cdot)}{q_{2}(\cdot)}}} \leq C 2^{-k_{0} \lambda_{2}} \sum_{k=0}^{k_{0}}\left(\left\|\frac{2^{k \alpha_{+}}\left|f \chi_{k}\right|}{\eta_{1}}\right\|_{L^{p_{1}(\cdot)}}\right)^{\left(q_{2}^{1}\right)_{k}} \\
& \leq C 2^{-k_{0} \lambda_{2}} \sum_{k=0}^{k_{0}}\left\|\left(\frac{2^{k \alpha_{+}}\left|f \chi_{k}\right|}{\eta_{1}}\right)^{q_{1}(\cdot)}\right\|_{L^{\frac{p_{1}(\cdot)}{q_{1}(\cdot)}}}^{\frac{\left(q_{2}^{1}\right)_{k}}{\left(q_{1}^{1}\right)_{j}}} \leq C \sum_{k=0}^{k_{0}}\left\{2^{-k_{0} \lambda_{1}}\left\|\left(\frac{2^{k \alpha_{+}}\left|f \chi_{k}\right|}{\eta_{1}}\right)^{q_{1}(\cdot)}\right\|_{L_{\frac{1}{q_{1}(\cdot)}}}\right\}^{\frac{\left(q_{2}^{1}\right)_{k}}{\left(q_{1}^{1}\right)_{j}}} \leq C,
\end{aligned}
$$


where

$$
\left(q_{1}^{1}\right)_{j}=\left\{\begin{array}{l|l}
\left(q_{1}\right)_{+}, & \| \frac{2^{k \alpha+}\left|f_{\chi_{k}}\right|}{\eta_{1}} \\
\left(q_{1}\right)_{-}, & \left\|\frac{2^{k \alpha_{+}}\left|f_{\chi_{k}}\right|}{\eta_{1}}\right\|_{L^{p_{1}(\cdot)}}>1
\end{array}\right.
$$

This implies that

$$
\eta_{12} \leq C \eta_{1} \leq C \sum_{|\gamma|=m}\left\|D^{\gamma} A\right\|_{B M O\left(\mathbb{R}^{n}\right)}\|f\|_{M K_{q_{1}(\cdot), p_{1}(\cdot)}^{\alpha(\cdot), \lambda_{1}}}\left(\mathbb{R}^{n}\right) .
$$

Now we estimate of $\eta_{11}$. Let $x \in C_{k} j \leq k-2$, then $|x-y| \sim|x|$, we can write that

$$
\left|T_{\Omega, \mu}^{A} f_{j}(x)\right| \leq C \int_{C_{j}} \frac{|\Omega(x-y)|}{|x-y|^{n-\mu+m}}\left|R_{m+1}(A ; x, y)\right|\left|f_{j}(y)\right| \mathrm{d} y,
$$

where

$$
A_{k}(x)=A(x)-\sum_{|\gamma|=m} \frac{1}{\gamma !}\left(D^{\gamma} A\right) x^{\gamma}
$$

and

$$
R_{m+1}(A ; x, y)=R_{m+1}\left(A_{k} ; x, y\right), D^{\gamma} A_{k}(x)=D^{\gamma} A(x)-m_{\gamma_{k}}\left(D^{\gamma} A\right),|\gamma|=m .
$$

Applying Lemma 10, we see that

$$
\begin{aligned}
\left|R_{m+1}(A ; x, y)\right| & =\left|R_{m+1}\left(A_{k} ; x, y\right)\right| \\
& \leq\left|R_{m}\left(A_{k} ; x, y\right)\right|+\sum_{|\gamma|=m} \frac{1}{\gamma !}\left|D^{\gamma} A_{k}(y)\right||x-y|^{m} \\
& \leq C|x-y|^{m} \sum_{|\gamma|=m}\left(\left(\frac{1}{|\widetilde{Q}(x, y)|} \int_{\tilde{Q}(x, y)}\left|D^{\gamma} A_{k}(z)\right|^{q} \mathrm{~d} z\right)^{\frac{1}{q}}+\frac{1}{\gamma !}\left|D^{\gamma} A_{k}(y)\right|\right) \\
& \leq C|x-y|^{m} \sum_{|\gamma|=m}\left(\left\|D^{\gamma} A\right\|_{B M O\left(\mathbb{R}^{n}\right)}+\left|D^{\gamma} A_{k}(y)\right|\right) .
\end{aligned}
$$

Thus, we get

$$
\begin{aligned}
\left|T_{\Omega, \mu}^{A} f_{j}(x)\right| & \leq C \int_{C_{j}} \frac{|\Omega(x-y)|}{|x-y|^{n-\mu}} \sum_{|\gamma|=m}\left[\left\|D^{\gamma} A\right\|_{B M O\left(\mathbb{R}^{n}\right)}+\left|D^{\gamma} A_{k}(y)\right|\right]\left|f_{j}(y)\right| \mathrm{d} y \\
& \leq C \sum_{|\gamma|=m} \int_{C_{j}} \frac{|\Omega(x-y)|}{|x-y|^{n-\mu}}\left[\left\|D^{\gamma} A\right\|_{B M O\left(\mathbb{R}^{n}\right)}+\left|D^{\gamma} A_{k}(y)\right|\right]\left|f_{j}(y)\right| \mathrm{d} y \\
& \leq C \sum_{|\gamma|=m}\left\|D^{\gamma} A\right\|_{B M O\left(\mathbb{R}^{n}\right)} \int_{C_{j}} \frac{|\Omega(x-y)|}{|x-y|^{n-\mu}\left|f_{j}(y)\right| \mathrm{d} y} \\
& +\sum_{|\gamma|=m} \int_{C_{j}} \frac{|\Omega(x-y)|}{|x-y|^{n-\mu}}\left|D^{\gamma} A_{k}(y)\right|\left|f_{j}(y)\right| \mathrm{d} y \\
& =: L_{1}+L_{2} .
\end{aligned}
$$

Applying the generalized Hölder's inequality, we have

$$
L_{1} \leq C \sum_{|\gamma|=m}\left\|D^{\gamma} A\right\|_{B M O\left(\mathbb{R}^{n}\right)}\left\|f_{j}\right\|_{L^{p_{1}(\cdot)}}\left\|\frac{\Omega(x-y)}{|x-y|^{n-\mu}} \chi_{j}\right\|_{L^{p^{\prime} 1(\cdot)}} .
$$

If $\frac{1}{\hat{p}_{1}(\cdot)}=\frac{1}{r}+\frac{1}{p_{1}^{\prime}(\cdot)}$, by Lemma 2, then we have

$$
L_{1} \leq \sum_{|\gamma|=m}\left\|D^{\gamma} A\right\|_{B M O\left(\mathbb{R}^{n}\right)}\left\|f_{j}\right\|_{L^{p_{1}(\cdot)}}\|\Omega(x-y)\|_{L^{r}}\left\|\frac{\chi_{j}}{|x-y|^{n-\mu}}\right\|_{L^{\tilde{p}_{1}^{\prime}(\cdot)}}
$$




$$
\begin{aligned}
& \leq C \sum_{|\gamma|=m}\left\|D^{\gamma} A\right\|_{B M O\left(\mathbb{R}^{n}\right)} 2^{-k(n-\mu)}\left\|f_{j}\right\|_{L^{p_{1}(\cdot)}}\left\|\chi_{j}\right\|_{L^{p_{1}^{\prime}(\cdot)}}\left[\int_{2^{k-2}}^{2^{k}} r^{n-1} \mathrm{~d} r \int_{S^{n-1}}\left|\Omega\left(y^{\prime}\right)\right|^{r} \mathrm{~d} \sigma\left(y^{\prime}\right)\right]^{\frac{1}{r}} \\
& \leq C \sum_{|\gamma|=m}\left\|D^{\gamma} A\right\|_{B M O\left(\mathbb{R}^{n}\right)} 2^{-k(n-\mu)}\left\|f_{j}\right\|_{L^{p_{1}(\cdot)}}\left\|\chi_{j}\right\|_{L^{\widetilde{p_{1}^{\prime}}(\cdot)}} 2^{\frac{k n}{r}}\|\Omega\|_{L^{r}\left(S^{n-1}\right)} \\
& \leq C \sum_{|\gamma|=m}\left\|D^{\gamma} A\right\|_{B M O\left(\mathbb{R}^{n}\right)} 2^{-k(n-\mu)} 2^{\frac{k n}{r}}\left\|f_{j}\right\|_{L^{p_{1}(\cdot)}}\left\|\chi_{B_{j}}\right\|_{L^{\widetilde{p_{1}^{\prime}}(\cdot)}} \cdot
\end{aligned}
$$

According to Lemma 3 and the formula $\frac{1}{p_{1}^{\prime}(\cdot)}=\frac{1}{p_{1}^{\prime}(\cdot)}-\frac{1}{r}$, we have

$$
\left\|\chi_{B_{j}}\right\|_{L_{p^{\prime}}^{p_{(}(\cdot)}} \approx\left\|\chi_{B_{j}}\right\|_{L^{p_{1}^{\prime}(\cdot)}}\left|B_{j}\right|^{\frac{-1}{r}}
$$

Then, we obtain

$$
L_{1} \leq \sum_{|\gamma|=m}\left\|D^{\gamma} A\right\|_{B M O\left(\mathbb{R}^{n}\right)} 2^{-k(n-\mu)} 2^{(k-j) \frac{n}{r}}\left\|f_{j}\right\|_{L^{p_{1}(\cdot)}}\left\|\chi_{B_{j}}\right\|_{L^{p_{1}^{\prime}(\cdot)}} .
$$

For $L_{2}$, applying the generalized Hölder's inequality, we get

$$
L_{2} \leq C \sum_{|\gamma|=m}\left\|f_{j}\right\|_{L^{p_{1}(\cdot)}}\left\|\frac{\Omega(x-y)}{|x-y|^{n-\mu}} \chi_{j}\left|D^{\gamma} A_{k}(y)\right|\right\|_{L^{p_{1}^{\prime}(\cdot)}} .
$$

If $\frac{1}{p_{1}^{\prime}(\cdot)}=\frac{1}{r}+\frac{1}{\bar{p}_{1}^{\prime}(\cdot)}$, by Lemma 3, we have

$$
\begin{aligned}
L_{2} & \leq C \sum_{|\gamma|=m} 2^{-k(n-\mu)}\left\|f_{j}\right\|_{L^{p_{1}(\cdot)}}\|\Omega(x-y)\|_{L^{r}}\left\|\chi_{j}\left|D^{\gamma} A_{k}(y)\right|\right\|_{L^{p_{1}^{\prime}(\cdot)}} \\
& \leq C 2^{-k(n-\mu)}\left\|f_{j}\right\|_{L^{p_{1}(\cdot)}}\|\Omega(x-y)\|_{L^{r}} \sum_{|\gamma|=m}\left\|\chi_{j}\left(D^{\gamma} A(x)-m_{\gamma_{k}}\left(D^{\gamma} A\right)\right)\right\|_{L^{\widetilde{p_{1}^{\prime}}(\cdot)}} .
\end{aligned}
$$

Similarly, and applying Lemma 7, we conclude that

$$
L_{2} \leq \sum_{|\gamma|=m}\left\|D^{\gamma} A\right\|_{B M O\left(\mathbb{R}^{n)}\right)}(k-j) 2^{-k(n-\mu)} 2^{(k-j) \frac{n}{r}}\left\|f_{j}\right\|_{L^{p_{1}(\cdot)}}\left\|\chi_{B_{j}}\right\|_{L^{p_{1}^{\prime}(\cdot)}} .
$$

Combining the above two estimates about $L_{1}, L_{2}$, we obtain

$$
\left|T_{\Omega, \mu}^{A} f_{j}(x)\right| \leq C \sum_{|\gamma|=m}\left\|D^{\gamma} A\right\|_{B M O\left(\mathbb{R}^{n}\right)}(k-j) 2^{-k(n-\mu)} 2^{(k-j) \frac{n}{r}}\left\|f_{j}\right\|_{L^{p_{1}(\cdot)}}\left\|\chi_{B_{j}}\right\|_{L^{p_{1}^{\prime}(\cdot)}} .
$$

From this and using Lemma 6, we deduce that

$$
\left.2^{-k_{0} \lambda_{2}} \sum_{k=0}^{k_{0}}\left\|\left(\frac{2^{k \alpha(\cdot)}\left|\sum_{j=0}^{k-2} T_{\Omega, \mu}^{A}\left(f_{j}\right) \chi_{k}\right|}{\eta_{1} \sum_{|\gamma|=m}\left\|D^{\gamma} A\right\|_{B M O\left(\mathbb{R}^{n}\right)}}\right)^{q_{2}(\cdot)}\right\|\right|_{L^{\frac{p_{2}(\cdot)}{q_{2}(\cdot)}}} \leq 2^{-k_{0} \lambda_{2}} \sum_{k=0}^{k_{0}}\left\|\frac{2^{k \alpha(\cdot)}\left|\sum_{j=0}^{k-2} T_{\Omega, \mu}^{A}\left(f_{j}\right) \chi_{k}\right|}{\eta_{1} \sum_{|\gamma|=m}\left\|D^{\gamma} A\right\|_{B M O\left(\mathbb{R}^{n}\right)}}\right\|_{L^{p_{2}(\cdot)}}^{\left(q_{2}^{2}\right)_{k}}
$$

where

$$
\left(q_{2}^{2}\right)_{k}= \begin{cases}\left(q_{2}\right)_{-}, & \left\|\left(\frac{2^{k \alpha(\cdot)}\left|\sum_{j=0}^{k-2} T_{\Omega, \mu}^{A}\left(f_{j}\right) \chi_{k}\right|}{\eta_{1} \sum_{|\gamma|=m}\left\|D^{\gamma} A\right\|_{B M O}\left(\mathbb{R}^{n}\right)}\right)^{q_{2}(\cdot)}\right\| \leq 1, \\ \left(q_{2}\right)_{+}, & \left\|\left(\frac{2^{k \alpha(\cdot) \mid} \sum_{j=0}^{k-2} T_{\Omega, \mu}^{A}\left(f_{j}\right) \chi_{k} \mid}{\eta_{1} \sum_{|\gamma|=m}\left\|D^{\gamma} A\right\|_{B M O}\left(\mathbb{R}^{n}\right)}\right)^{q_{2}(\cdot)}\right\|_{L^{p_{2}(\cdot)}}>1 .\end{cases}
$$


Noting that if $\frac{1}{p_{1}(\cdot)}-\frac{1}{p_{2}(\cdot)}=\frac{\mu}{n}$, then $C_{1}|B|^{\frac{\mu}{n}}\left\|\chi_{B}\right\|_{L^{p_{2}(\cdot)}} \leq\left\|\chi_{B}\right\|_{L^{p_{1}(\cdot)}} \leq C_{2}|B|^{\frac{\mu}{n}}\left\|\chi_{B}\right\|_{L^{p_{2}(\cdot)}}$ (see[22], p.370).

Therefore, together with this and applying Lemma 4 and Lemma 5, we have

$$
\begin{aligned}
& 2^{-k_{0} \lambda_{2}} \sum_{k=0}^{k_{0}}\left\|\left(\frac{2^{k \alpha(\cdot)}\left|\sum_{j=0}^{k-2} T_{\Omega, \mu}^{A}\left(f_{j}\right) \chi_{k}\right|}{\eta_{1} \sum_{|\gamma|=m}\left\|D^{\gamma} A\right\|_{B M O\left(\mathbb{R}^{n}\right)}}\right)^{q_{2}(\cdot)}\right\|_{L^{\frac{p_{2}(\cdot)}{q_{2}(\cdot)}}} \\
& \leq 2^{-k_{0} \lambda_{2}} \sum_{k=0}^{k_{0}}\left[2^{k \alpha(\cdot)} \sum_{j=0}^{k-2} 2^{-k(n-\mu)} 2^{(k-j) \frac{n}{r}}(k-j)\left\|\frac{\left|f_{j}\right|}{\eta_{1}}\right\|_{L^{p_{1}(\cdot)}\left(\mathbb{R}^{n}\right)}\left\|\chi_{B_{j}}\right\|_{L^{p_{1}^{\prime}(\cdot)}}\left\|\chi_{B_{k}}\right\|_{L^{p_{2}(\cdot)}}\right]^{\left(q_{2}^{2}\right)_{k}} \\
& \leq C 2^{-k_{0} \lambda_{2}} \sum_{k=0}^{k_{0}}\left[2^{k \alpha(\cdot)} \sum_{j=0}^{k-2} 2^{-k(n-\mu)} 2^{(k-j) \frac{n}{r}}(k-j)\left\|\frac{\left|f_{j}\right|}{\eta_{1}}\right\|_{L^{p_{1}(\cdot)}\left(\mathbb{R}^{n}\right)}\left\|\chi_{B_{j}}\right\|_{L^{p_{1}^{\prime}(\cdot)}}\left|B_{k}\right|^{-\frac{\mu}{n}}\left\|\chi_{B_{k}}\right\|_{L^{p_{1}(\cdot)}}\right]^{\left(q_{2}^{2}\right)_{k}} \\
& \leq C 2^{-k_{0} \lambda_{2}} \sum_{k=-\infty}^{\infty}\left[2^{k \alpha(\cdot)} \sum_{j=0}^{k-2}(k-j) 2^{(k-j) \frac{n}{r}}\left\|\frac{\left|f_{j}\right|}{\eta_{1}}\right\|_{L^{p_{1}(\cdot)}\left(\mathbb{R}^{n}\right)} \frac{\left\|\chi_{B_{j}}\right\|_{L_{1}^{p_{1}^{\prime}(\cdot)}}}{\left\|\chi_{B_{k}}\right\|_{L^{p_{1}^{\prime}(\cdot)}}}\right]^{\left(q_{2}^{2}\right)_{k}} \\
& \leq C 2^{-k_{0} \lambda_{2}} \sum_{k=0}^{\infty}\left[2^{k \alpha(\cdot)} \sum_{j=0}^{k-2}(k-j) 2^{(j-k)\left(n \delta_{1}-\frac{n}{r}\right)}\left\|\frac{\left|f_{j}\right|}{\eta_{1}}\right\|_{L^{p_{1}(\cdot)}\left(\mathbb{R}^{n}\right)}\right]^{\left(q_{2}^{2}\right)_{k}} \\
& \left.\leq C 2^{-k_{0} \lambda_{2}} \sum_{k=0}^{\infty}\left[\sum_{j=0}^{k-2}(k-j) 2^{(j-k)\left(n \delta_{1}-\frac{n}{r}-\alpha_{+}\right.}\right)\left\|\frac{2^{j \alpha_{+}}\left|f \chi_{k}\right|}{\eta_{1}}\right\|_{L^{p_{1}(\cdot)}\left(\mathbb{R}^{n}\right)}\right]^{\left(q_{2}^{2}\right)_{k}} .
\end{aligned}
$$

Since $f \in M K_{q_{1}(\cdot), p_{1}(\cdot)}^{\alpha+, \lambda_{1}}\left(\mathbb{R}^{n}\right),\left(\lambda_{2}\right) /\left(q_{2}\right)_{+}=\left(\lambda_{1}\right) /\left(q_{1}\right)_{-}$and $\alpha_{+}<n \delta_{1}+\frac{n}{r}+\left(\lambda_{1}\right) /\left(q_{1}\right)_{-}$, we have

$$
\begin{aligned}
& 2^{-k_{0} \lambda_{2}} \sum_{k=0}^{k_{0}}\left\|\left(\frac{2^{k \alpha(\cdot)}\left|\sum_{j=0}^{k-2} T_{\Omega, \mu}^{A}\left(f_{j}\right) \chi_{k}\right|}{\eta_{1} \sum_{|\gamma|=m}\left\|D^{\gamma} A\right\|_{B M O\left(\mathbb{R}^{n}\right)}}\right)^{q_{2}(\cdot)}\right\|_{L^{\frac{p_{2}(\cdot)}{q_{2}(\cdot)}}} \\
& \left.\leq C 2^{-k_{0} \lambda_{2}} \sum_{k=0}^{\infty}\left[\sum_{j=0}^{k-2}(k-j) 2^{(j-k)\left(n \delta_{1}-\frac{n}{r}-\alpha_{+}\right.}\right)\left\|\left(\frac{2^{j \alpha_{+}}\left|f \chi_{k}\right|}{\eta_{1}}\right)^{q_{1}(\cdot)}\right\| \|_{L^{\frac{1}{q_{1}(\cdot)}}\left(\mathbb{R}^{n}\right)}^{\frac{1}{\left(q_{1}^{2}\right) j}}\right]^{\left(q_{2}^{2}\right)_{k}} \\
& \left.\leq C 2^{-k_{0} \lambda_{2}} \sum_{k=0}^{\infty}\left[\sum_{j=0}^{k-2}(k-j) 2^{(j-k)\left(n \delta_{1}-\frac{n}{r}-\alpha_{+}\right.}\right)\left(2^{j \lambda} 2^{-j \lambda} \sum_{n=0}^{j}\left\|\left(\frac{2^{n \alpha_{+}}\left|f \chi_{n}\right|}{\eta_{1}}\right)^{q_{1}(\cdot)}\right\|_{L^{\frac{p_{1}(\cdot)}{q_{1}(\cdot)}}\left(\mathbb{R}^{n}\right)}\right)^{\frac{1}{\left(q_{1}^{2}\right) j}}\right]^{\left(q_{2}^{2}\right)_{k}} \\
& \leq C \sum_{k=0}^{\infty} 2^{\left(k-k_{0}\right) \lambda_{2}} \\
& \times\left[\sum_{j=0}^{k-2}(k-j) 2^{(j-k)\left(\left(\lambda_{1}\right) /\left(q_{1}\right)_{-}+n \delta_{1}-\frac{n}{r}-\alpha_{+}\right)}\left(2^{-j \lambda} \sum_{n=0}^{j}\left\|\left(\frac{2^{n \alpha_{+}}\left|f \chi_{n}\right|}{\eta_{1}}\right)^{q_{1}(\cdot)}\right\|_{L^{\frac{p_{1}(\cdot)}{q_{1}(\cdot)}\left(\mathbb{R}^{n}\right)}}\right)^{\frac{1}{\left(q_{1}^{2}\right) j}}\right]^{\left(q_{2}^{2}\right)_{k}} \\
& \leq C \sum_{k=0}^{\infty} 2^{\left(k-k_{0}\right) \lambda_{2}}\left[\sum_{j=0}^{k-2}(k-j) 2^{(j-k)\left(\left(\lambda_{1}\right) /\left(q_{1}\right)_{-}+n \delta_{1}-\frac{n}{r}-\alpha_{+}\right)}\right]^{\left(q_{2}^{2}\right)_{k}} \leq C,
\end{aligned}
$$

where

$$
\left(q_{1}^{2}\right)_{j}=\left\{\begin{array}{l|l}
\left(q_{1}\right)_{-}, & \| \frac{2^{j \alpha_{+}}\left|f_{\chi_{j}}\right|}{\eta_{1}} \\
\left(q_{1}\right)_{+}, & \left\|\frac{2^{j^{\alpha+}}\left|f_{\chi_{j}}\right|}{\eta_{1}}\right\|_{L^{p_{1}(\cdot)}} \leq 1, \\
L^{p_{1}(\cdot)} & >1 .
\end{array}\right.
$$


This implies that

$$
\eta_{11} \leq C \eta_{1} \sum_{|\gamma|=m}\left\|D^{\gamma} A\right\|_{B M O\left(\mathbb{R}^{n}\right)}=\sum_{|\gamma|=m}\left\|D^{\gamma} A\right\|_{B M O\left(\mathbb{R}^{n}\right)}\|f\|_{M K_{q_{1}(\cdot), p_{1}(\cdot)}^{\alpha(\cdot), \lambda_{1}}\left(\mathbb{R}^{n}\right)} .
$$

Finally, we estimate of $\eta_{13}$. Let $x \in C_{k}, j \geq k+2$, then $|x-y| \sim|y|$, by an argument similar to used in $\eta_{11}$, we have

$$
\left|T_{\Omega, \mu}^{A} f_{j}(x)\right| \leq C \sum_{|\gamma|=m}\left\|D^{\gamma} A\right\|_{B M O\left(\mathbb{R}^{n}\right)}(k-j) 2^{-j(n-\mu)}\left\|f_{j}\right\|_{L^{p_{1}(\cdot)}}\left\|\chi_{B_{j}}\right\|_{L^{p_{1}^{\prime}(\cdot)}} .
$$

Applying Lemma 6, we have

$$
2^{-k_{0} \lambda_{2}} \sum_{k=0}^{k_{0}}\left\|\left(\frac{2^{k \alpha(\cdot)}\left|\sum_{k+2}^{\infty} T_{\Omega, \mu}^{A}\left(f_{j}\right) \chi_{k}\right|}{\eta_{1} \sum_{|\gamma|=m}\left\|D^{\gamma} A\right\|_{B M O\left(\mathbb{R}^{n}\right)}}\right)^{q_{2}(\cdot)}\right\|_{L^{\frac{p_{2}(\cdot)}{q_{2}(\cdot)}}} \leq 2^{-k_{0} \lambda_{2}} \sum_{k=0}^{k_{0}}\left\|\frac{2^{k \alpha(\cdot)}\left|\sum_{j=k+2}^{\infty} T_{\Omega, \mu}^{A}\left(f_{j}\right) \chi_{k}\right|}{\eta_{1} \sum_{|\gamma|=m}\left\|D^{\gamma} A\right\|_{B M O\left(\mathbb{R}^{n}\right)}}\right\|_{L^{p_{2}(\cdot)}}^{\left(q_{2}^{3}\right)_{k}}
$$

where

$$
\left(q_{2}^{3}\right)_{k}=\left\{\begin{array}{l|l}
\left(q_{2}\right)_{-}, & \left\|\left(\frac{2^{k \alpha(\cdot) \mid} \sum_{j=k+2}^{\infty} T_{\Omega, \mu}^{A}\left(f_{j}\right) \chi_{k} \mid}{\eta_{1} \sum|\gamma|=m\left\|D^{\gamma} A\right\|_{B M O\left(\mathbb{R}^{n}\right)}}\right)^{q_{2}(\cdot)}\right\|_{L^{p_{2}(\cdot)}} \leq 1, \\
\left(q_{2}\right)_{+}, & \left\|\left(\frac{2^{k \alpha(\cdot) \mid} \sum_{j=k+2}^{\infty} T_{\Omega, \mu}^{A}\left(f_{j}\right) \chi_{k} \mid}{\eta_{1} \sum_{|\gamma|=m}\left\|D^{\gamma} A\right\|_{B M O}\left(\mathbb{R}^{n}\right)}\right)^{q_{2}(\cdot)}\right\|_{L^{p_{2}(\cdot)}}>1 .
\end{array}\right.
$$

Therefore, applying Lemma 4 and Lemma 5, we get

$$
\begin{aligned}
& 2^{-k_{0} \lambda_{2}} \sum_{k=0}^{k_{0}}\left[2^{k \alpha(\cdot)} \sum_{j=k+2}^{\infty} 2^{-j(n-\mu)}(k-j)\left\|\frac{\left|f_{j}\right|}{\eta_{1}}\right\|_{L^{p_{1}(\cdot)\left(\mathbb{R}^{n}\right)}}\left\|\chi_{B_{j}}\right\|_{L^{p_{1}^{\prime}(\cdot)}}\left\|\chi_{B_{k}}\right\|_{L^{p_{2}(\cdot)}}\right]^{\left(q_{2}^{3}\right)_{k}} \\
& \leq C 2^{-k_{0} \lambda_{2}} \sum_{k=0}^{k_{0}}\left[2^{k \alpha(\cdot)} \sum_{j=k+2}^{\infty} 2^{-j(n-\mu)}(k-j)\left\|\frac{\left|f_{j}\right|}{\eta_{1}}\right\|_{L^{p_{1}(\cdot)\left(\mathbb{R}^{n}\right)}}\left\|\chi_{B_{j}}\right\|_{L^{p_{1}^{\prime}(\cdot)}} 2^{-\mu k}\left\|\chi_{B_{k}}\right\|_{L^{p_{1}(\cdot)}}\right]^{\left(q_{2}^{3}\right)_{k}} \\
& \leq C 2^{-k_{0} \lambda_{2}} \sum_{k=0}^{k_{0}}\left[2^{k \alpha(\cdot)} \sum_{j=k+2}^{\infty} 2^{(j-k) \mu}(k-j)\left\|\frac{\left|f_{j}\right|}{\eta_{1}}\right\|_{L^{p_{1}(\cdot)}\left(\mathbb{R}^{n}\right)} 2^{-j n}\left\|\chi_{B_{j}}\right\|_{L^{p_{1}^{\prime}(\cdot)}}\left\|\chi_{B_{k}}\right\|_{L^{p_{1}(\cdot)}}\right]^{\left(q_{2}^{3}\right)_{k}} \\
& \leq C 2^{-k_{0} \lambda_{2}} \sum_{k=0}^{k_{0}}\left[2^{k \alpha(\cdot)} \sum_{j=k+2}^{\infty} 2^{(j-k) \mu}(k-j)\left\|\frac{\left|f_{j}\right|}{\eta_{1}}\right\|_{L^{p_{1}(\cdot)}\left(\mathbb{R}^{n}\right)} \frac{\left\|\chi_{B_{k}}\right\|_{L^{p_{1}(\cdot)}}}{\left\|\chi_{B_{j}}\right\|_{L^{p_{1}(\cdot)}}}\right]^{\left(q_{2}^{3}\right)_{k}} \\
& \leq C 2^{-k_{0} \lambda_{2}} \sum_{k=0}^{k_{0}}\left[2^{k \alpha(\cdot)} \sum_{j=k+2}^{\infty}(k-j) 2^{(k-j)\left(n \delta_{2}-\mu\right)}\left\|\frac{\left|f_{j}\right|}{\eta_{1}}\right\|_{L^{p_{1}(\cdot)}\left(\mathbb{R}^{n}\right)}\right]^{\left(q_{2}^{3}\right)_{k}} \text {. }
\end{aligned}
$$

Notice that $f \in M K_{q_{1}(\cdot), p_{1}(\cdot)}^{\alpha_{+}, \lambda_{1}}\left(\mathbb{R}^{n}\right),\left(\lambda_{2}\right) /\left(q_{2}\right)_{+}=\left(\lambda_{1}\right) /\left(q_{1}\right)_{-}$and $\alpha_{+}>\mu-n \delta_{2}+\left(\lambda_{1}\right) /\left(q_{1}\right)_{-}$, we have

$$
\begin{aligned}
& 2^{-k_{0} \lambda_{2}} \sum_{k=0}^{k_{0}}\left\|\left(\frac{2^{k \alpha(\cdot)}\left|\sum_{j=k+2}^{\infty} T_{\Omega, \mu}^{A}\left(f_{j}\right) \chi_{k}\right|}{\eta_{1} \sum_{|\gamma|=m}\left\|D^{\gamma} A\right\|_{B M O\left(\mathbb{R}^{n}\right)}}\right)^{q_{2}(\cdot)}\right\|_{L^{\frac{p_{2}(\cdot)}{q_{2} \cdot(\cdot)}}} \\
& \leq C 2^{-k_{0} \lambda_{2}} \sum_{k=0}^{\infty}\left[\sum_{j=k+2}^{\infty}(k-j) 2^{(k-j)\left(n \delta_{2}-\mu+\alpha_{+}\right)}\left\|\left(\frac{2^{j \alpha_{+}}\left|f \chi_{k}\right|}{\eta_{1}}\right)^{q_{1}(\cdot)}\right\|_{L^{\frac{1}{q_{1}^{3}(\cdot)}}\left(\mathbb{R}^{n}\right)}^{\left(q_{1}^{3}\right)}\right]^{\left(q_{2}^{3}\right)_{k}}
\end{aligned}
$$




$$
\begin{aligned}
& \left.\leq C 2^{-k_{0} \lambda_{2}} \sum_{k=0}^{\infty}\left[\sum_{j=k+2}^{\infty}(k-j) 2^{(k-j)\left(n \delta_{2}-\mu+\alpha_{+}\right.}\right)\left(2^{j \lambda} 2^{-j \lambda} \sum_{n=0}^{j}\left\|\left(\frac{2^{n \alpha_{+}}\left|f \chi_{n}\right|}{\eta_{1}}\right)^{q_{1}(\cdot)}\right\|_{L^{\frac{p_{1}(\cdot)}{q_{1}(\cdot)}\left(\mathbb{R}^{n}\right)}}\right)^{\frac{1}{\left(q_{1}^{3}\right) j}}\right]^{\left(q_{2}^{3}\right)_{k}} \\
& \leq C \sum_{k=0}^{\infty} 2^{\left(k-k_{0}\right) \lambda_{2}} \\
& \times\left[\sum_{j=k+2}^{\infty}(k-j) 2^{(k-j)\left(n \delta_{2}-\mu-\left(\lambda_{1}\right) /\left(q_{1}\right)_{-}+\alpha_{+}\right)}\left(2^{-j \lambda} \sum_{n=0}^{j}\left\|\left(\frac{2^{n \alpha_{+}}\left|f \chi_{n}\right|}{\eta_{1}}\right)^{q_{1}(\cdot)}\right\|_{L^{\frac{p_{1}(\cdot)}{q_{1} \cdot(\cdot)}\left(\mathbb{R}^{n}\right)}}\right)^{\frac{1}{\left(q_{1}^{3}\right) j}}\right]^{\left(q_{2}^{3}\right)_{k}} \\
& \leq C \sum_{k=0}^{\infty} 2^{\left(k-k_{0}\right) \lambda_{2}}\left[\sum_{j=k+2}^{\infty}(k-j) 2^{(k-j)\left(n \delta_{2}-\mu-\left(\lambda_{1}\right) /\left(q_{1}\right)_{-}-\alpha_{+}\right)}\right]^{\left(q_{2}^{3}\right)_{k}} \leq C,
\end{aligned}
$$

where

$$
\left(q_{1}^{3}\right)_{j}=\left\{\begin{array}{l|l}
\left(q_{1}\right)_{-}, & \| \frac{2^{j \alpha_{+}}\left|f_{x_{j}}\right|}{\eta_{1}} \\
\left(q_{1}\right)_{+}, & \left\|\frac{2^{j \alpha_{+}}\left|f_{\chi_{j}}\right|}{\eta_{1}}\right\|_{L^{p_{1}(\cdot)}} \leq 1 \\
L^{p_{1}(\cdot)} & >1 .
\end{array}\right.
$$

This implies that

$$
\eta_{13} \leq C \eta_{1} \sum_{|\gamma|=m}\left\|D^{\gamma} A\right\|_{B M O\left(\mathbb{R}^{n}\right)}=\sum_{|\gamma|=m}\left\|D^{\gamma} A\right\|_{B M O\left(\mathbb{R}^{n}\right)}\|f\|_{M K_{q_{1}(\cdot), p_{1}(\cdot)}^{\alpha(\cdot), \lambda_{1}}\left(\mathbb{R}^{n}\right)} .
$$

The proof of Theorem 1 is finished.

Theorem 2. Suppose that $D^{\gamma} A \in \dot{\Lambda}_{\beta}(|\gamma|=|m|, m \geq 1)$. Let $0<\mu<n, \Omega \in L^{r}\left(s^{n-1}\right), q_{1}(\cdot), q_{2}(\cdot) \in \mathcal{P}\left(\mathbb{R}^{n}\right)$ with $\left(q_{2}\right)_{-} \geq\left(q_{1}\right)_{+}$, and $p_{1}(\cdot), p_{2}(\cdot) \in \mathfrak{B}\left(\mathbb{R}^{n}\right)$ satisfy $0<\mu+\beta \leq \frac{n}{\left(p_{1}\right)_{+}}, \frac{1}{p_{1}(x)}-\frac{1}{p_{2}(x)}=\frac{\mu+\beta}{n}$. If $\left(\lambda_{1}\right)\left(q_{2}\right)_{+}=\left(\lambda_{2}\right)\left(q_{1}\right)_{-}$and $(\mu+\beta)-n \delta_{2}+\left(\lambda_{1}\right) /\left(q_{1}\right)_{-}<\alpha_{+}<n \delta_{1}+\frac{n}{r}+\left(\lambda_{1}\right) /\left(q_{1}\right)_{-}$. Then $T_{\Omega, \mu}^{A}$ is bounded from $M K_{q_{1}(\cdot), p_{1}(\cdot)}^{\alpha_{+}, \lambda_{1}}\left(\mathbb{R}^{n}\right)$ to $M K_{q_{2}(\cdot), p_{2}(\cdot)}^{\alpha(\cdot), \lambda_{2}}\left(\mathbb{R}^{n}\right)$.

Proof. Let $D^{\gamma} A \in \Lambda_{\beta}\left(\mathbb{R}^{n}\right), f \in M K_{q_{1}(\cdot), p_{1}(\cdot)}^{\alpha_{+}, \lambda_{1}}\left(\mathbb{R}^{n}\right)$, we write

$$
f(x)=\sum_{j=0}^{\infty} f(x) \chi_{j}=\sum_{j=0}^{\infty} f_{j}(x) .
$$

By the definition of $M K_{q(\cdot), p(\cdot)}^{\alpha(\cdot),}\left(\mathbb{R}^{n}\right)$, we have

$$
\left\|T_{\Omega, \mu}^{A}(f) \chi_{k}\right\|_{M K_{q_{2}(\cdot), p_{2}(\cdot)}^{\alpha(\cdot), \lambda_{2}}\left(\mathbb{R}^{n}\right)}=\inf \left\{\eta>0: \sup _{k_{0} \in z} 2^{-k_{0} \lambda_{2}} \sum_{k=0}^{k_{0}}\left\|\left(\frac{2^{k \alpha(\cdot)}\left|T_{\Omega, \mu}^{A}(f) \chi_{k}\right|}{\eta}\right)^{q_{2}(\cdot)}\right\|_{L_{L_{2}(\cdot)}^{p_{2}(\cdot)}} \leq 1\right\} .
$$

For any $k_{0} \in z$, we see that

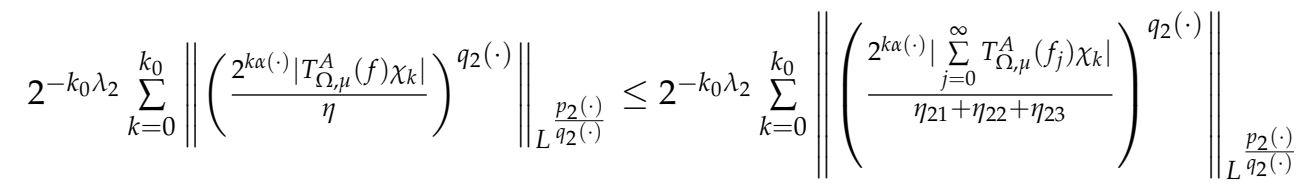

$$
\begin{aligned}
& \leq 2^{-k_{0} \lambda_{2}} \sum_{k=0}^{k_{0}}\left\|\left(\frac{2^{k \alpha(\cdot)}\left|\sum_{j=0}^{k-2} T_{\Omega, \mu}^{A}\left(f_{j}\right) \chi_{k}\right|}{\eta_{21}}\right)^{q_{2}(\cdot)}\right\|_{L^{\frac{p_{2}(\cdot)}{q_{2}(\cdot)}}}+2^{-k_{0} \lambda_{2}} \sum_{k=0}^{k_{0}}\left\|\left(\frac{2^{k \alpha(\cdot)}\left|\sum_{j=k-1}^{k+1} T_{\Omega, \mu}^{A}\left(f_{j}\right) \chi_{k}\right|}{\eta_{22}}\right)^{q_{2}(\cdot)}\right\|_{L^{\frac{p_{2}(\cdot)}{q_{2}(\cdot)}}} \\
& +2^{-k_{0} \lambda_{2}} \sum_{k=0}^{k_{0}}\left\|\left(\frac{2^{k \alpha(\cdot) \mid} \sum_{j=k+2}^{\infty} T_{\Omega, \mu}^{A}\left(f_{j}\right) \chi_{k} \mid}{\eta_{23}}\right)^{q_{2}(\cdot)}\right\|_{L^{\frac{p_{2}(\cdot)}{q_{2}(\cdot)}}},
\end{aligned}
$$


where

$$
\begin{aligned}
& \eta_{21}=\left\|\sum_{j=0}^{k-2} T_{\Omega, \mu}^{A}\left(f_{j}\right) \chi_{k}\right\|_{M K_{q_{2}(\cdot), p_{2}(\cdot)}^{\alpha(\cdot) \lambda_{2}}\left(\mathbb{R}^{n}\right)} \\
& =\inf \left\{\eta>0: \sup _{k_{0} \in z} 2^{-k_{0} \lambda_{2}} \sum_{k=0}^{k_{0}}\left\|\left(\frac{2^{k \alpha(\cdot)}\left|\sum_{j=0}^{k-2} T_{\Omega, \mu}^{A}\left(f_{j}\right) \chi_{k}\right|}{\eta}\right)^{q_{2}(\cdot)}\right\|_{L^{\frac{p_{2}(\cdot)}{q_{2}(\cdot)}}} \leq 1\right\} \text {, } \\
& \eta_{22}=\left\|\sum_{k-1}^{k+1} T_{\Omega, \mu}^{A}\left(f_{j}\right) \chi_{k}\right\|_{M K_{q_{2}(\cdot), p_{2}(\cdot)}^{\alpha(\cdot), \lambda_{2}}\left(\mathbb{R}^{n}\right)} \\
& =\inf \left\{\eta>0: \sup _{k_{0} \in z} 2^{-k_{0} \lambda_{2}} \sum_{k=0}^{k_{0}}\left\|\left(\frac{2^{k \alpha(\cdot)}\left|\sum_{j=k-1}^{k+1} T_{\Omega, \mu}^{A}\left(f_{j}\right) \chi_{k}\right|}{\eta}\right)^{q_{2}(\cdot)}\right\|_{L_{\frac{p_{2}(\cdot)}{q_{2}(\cdot)}}} \leq 1\right\}, \\
& \eta_{23}=\left\|\sum_{j=k+2}^{\infty} T_{\Omega, \mu}^{A}\left(f_{j}\right) \chi_{k}\right\|_{M K_{q_{2}(\cdot), p_{2}(\cdot)}^{\alpha(\cdot), \lambda_{2}}\left(\mathbb{R}^{n}\right)} \\
& =\inf \left\{\eta>0: \sup _{k_{0} \in z} 2^{-k_{0} \lambda_{2}} \sum_{k=0}^{k_{0}}\left\|\left(\frac{2^{k \alpha(\cdot)}\left|\sum_{j=k+2}^{\infty} T_{\Omega, \mu}^{A}\left(f_{j}\right) \chi_{k}\right|}{\eta}\right)^{q_{2}(\cdot)}\right\|_{L_{\frac{p_{2}(\cdot)}{q_{2}(\cdot)}}} \leq 1\right\} .
\end{aligned}
$$

And $\eta=\eta_{21}+\eta_{22}+\eta_{23}$, thus

$$
2^{-k_{0} \lambda_{2}} \sum_{k=0}^{k_{0}}\left\|\left(\frac{2^{k \alpha(\cdot)}\left|T_{\Omega, \mu}^{A}\left(f_{j}\right) \chi_{k}\right|}{\eta}\right)^{q_{2}(\cdot)}\right\|_{L^{\frac{p_{2}(\cdot)}{q_{2}(\cdot)}}} \leq C .
$$

So, we have

$$
\left\|T_{\Omega, \mu}^{A}(f) \chi_{k}\right\|_{M K_{q_{2}(\cdot), p_{2}(\cdot)}^{\alpha(\cdot), \lambda_{2}}\left(\mathbb{R}^{n}\right)} \leq C \eta \leq C\left[\eta_{21}+\eta_{22}+\eta_{23}\right] .
$$

Hence

$$
\eta_{21}, \eta_{22}, \eta_{23} \leq C \sum_{|\gamma|=m}\left\|D^{\gamma} A\right\|_{\lambda_{\beta}}\|f\|_{M K_{q_{1}(\cdot), p_{1}(\cdot)}^{\alpha(\cdot), \lambda_{1}}\left(\mathbb{R}^{n}\right)}
$$

Denote $\eta_{1}=\|f\|_{M K_{q_{1}(\cdot), p_{1}(\cdot)}^{\alpha(\cdot)}}\left(\mathbb{R}^{n}\right) \cdot$

Now we consider $\eta_{22}$ firstly. Noting that $T_{\Omega, \mu}^{A}$ is bounded on $L^{p(\cdot)}$ ( Theorem 5 in [14]), as argued about $\eta_{12}$ in proof of Theorem 1, we can get

$$
2^{-k_{0} \lambda_{2}} \sum_{k=0}^{k_{0}}\left\|\left(\frac{2^{k \alpha(\cdot)}\left|\sum_{j=k-1}^{k+1} T_{\Omega, \mu}^{A}\left(f_{j}\right) \chi_{k}\right|}{\eta_{1} \sum_{|\gamma|=m}\left\|D^{\gamma} A\right\|_{\lambda_{\beta}}}\right)^{q_{2}(\cdot)}\right\|_{\frac{p_{2}(\cdot)}{q_{2}(\cdot)}} \leq C .
$$

This implies that

$$
\eta_{22} \leq C \eta_{1} \leq C \sum_{|\gamma|=m}\left\|D^{\gamma} A\right\|_{\Lambda_{\beta}}\|f\|_{M K_{q_{1}(\cdot), p_{1}(\cdot)}^{\alpha(\cdot), \lambda_{1}}\left(\mathbb{R}^{n}\right)} .
$$


Next, we consider $\eta_{21}$. Let $x \in C_{k}, j \leq k-2$, then $|x-y| \sim|x|$, we get

$$
\left|T_{\Omega, \mu}^{A} f_{j}(x)\right| \leq \int_{C_{j}} \frac{\left|\Omega(x-y) f_{j}(y)\right|}{|x-y|^{n-\mu+m}}\left|R_{m+1}(A ; x, y)\right| \mathrm{d} y .
$$

By Lemma 9, and applying Hölder's inequality, we have

$$
\begin{aligned}
\left|T_{\Omega, \mu}^{A} f_{j}(x)\right| & \leq C \sum_{|\gamma|=m}\left\|D^{\gamma} A\right\|_{\lambda_{\beta}} \int_{C_{j}} \frac{\left|\Omega(x-y) f_{j}(y)\right|}{|x-y|^{n-(\mu+\beta)}} \mathrm{d} y \\
& \leq C \sum_{|\gamma|=m}\left\|D^{\gamma} A\right\|_{\lambda_{\beta}}\left\|f_{j}\right\|_{L^{p_{1}(\cdot)}}\left\|\frac{\Omega(x-y)}{|x-y|^{n-(\mu+\beta)}} \chi_{j}\right\|_{L^{p^{\prime}{ }_{1}(\cdot)}} .
\end{aligned}
$$

In the same way as we estimated $L_{1}$ in Theorem 1 , we obtain that

$$
\left|T_{\Omega, \mu}^{A} f_{j}(x)\right| \leq C \sum_{|\gamma|=m}\left\|D^{\gamma} A\right\|_{\lambda_{\beta}} 2^{-k(n-(\mu+\beta))} 2^{(k-j) \frac{n}{r}}\left\|f_{j}\right\|_{L^{p_{1}(\cdot)}}\left\|\chi_{B_{j}}\right\|_{L^{p_{1}^{\prime}(\cdot)}} .
$$

Applying Lemma 6, we get that

$$
2^{-k_{0} \lambda_{2}} \sum_{k=0}^{k_{0}}\left\|\left(\frac{2^{k \alpha(\cdot)}\left|\sum_{j=0}^{k-2} T_{\Omega, \mu}^{A}\left(f_{j}\right) \chi_{k}\right|}{\eta_{1} \sum_{|\gamma|=m}\left\|D^{\gamma} A\right\|_{\lambda_{\beta}}}\right)^{q_{2}(\cdot)}\right\| 2_{L^{-p_{2}(\cdot)}} \leq \sum_{k=0}^{k_{2}(\cdot)}\left\|\frac{2^{k \alpha(\cdot)}\left|\sum_{j=0}^{k-2} T_{\Omega, \mu}^{A}\left(f_{j}\right) \chi_{k}\right|}{\eta_{1} \sum_{|\gamma|=m}\left\|D^{\gamma} A\right\|_{\lambda_{\beta}}}\right\| \|_{L^{p_{2}(\cdot)}}^{\left(q_{2}^{1}\right)_{k}} .
$$

Where

$$
\left(q_{2}^{1}\right)_{k}=\left\{\begin{array}{l|l}
\left(q_{2}\right)_{-}, & \left\|\left(\frac{2^{k \alpha(\cdot)}\left|\sum_{j=0}^{k-2} T_{\Omega, \mu}^{A}\left(f_{j}\right) x_{k}\right|}{\eta_{1} \sum_{|\gamma|=m}|| D^{\gamma} A \|_{\lambda_{\beta}}}\right)^{q_{2}(\cdot)}\right\|_{L^{p_{2}(\cdot)}} \leq 1, \\
\left(q_{2}\right)_{+}, & \left\|\left(\frac{2^{k \alpha(\cdot) \mid} \sum_{j=0}^{k-2} T_{\Omega, \mu}^{A}\left(f_{j}\right) x_{k} \mid}{\eta_{1} \sum_{|\gamma|=m}\left\|D^{\gamma} A\right\|_{\lambda_{\beta}}}\right)^{q_{2}(\cdot)}\right\|_{L^{p_{2}(\cdot)}}>1 .
\end{array}\right.
$$

Since $\frac{1}{p_{1}(\cdot)}-\frac{1}{p_{2}(\cdot)}=\frac{\mu+\beta}{n}$, then $\left\|\chi_{B}\right\|_{L^{p_{2}(\cdot)}} \leq C 2^{-k(\mu+\beta)}\left\|\chi_{B}\right\|_{L^{p_{1}(\cdot)}}$ (see[22], P. 370). Therefore, applying Lemma 4 and Lemma 5, we deduce that

$$
\begin{aligned}
& 2^{-k_{0} \lambda_{2}} \sum_{k=0}^{k_{0}}\left\|\left(\frac{2^{k \alpha(\cdot)}\left|\sum_{j=0}^{k-2} T_{\Omega, \mu}^{A}\left(f_{j}\right) \chi_{k}\right|}{\eta_{1} \sum_{|\gamma|=m}\left\|D^{\gamma} A\right\|_{\wedge_{\beta}}}\right)^{q_{2}(\cdot)}\right\|_{L^{\frac{p_{2}(\cdot)}{q_{2}(\cdot)}}} \\
& \leq C 2^{-k_{0} \lambda_{2}} \sum_{k=0}^{k_{0}}\left[2^{k \alpha(\cdot)} \sum_{j=0}^{k-2} 2^{-k(n-(\mu+\beta))} 2^{(k-j) \frac{n}{r}}\left\|\frac{\left|f_{j}\right|}{\eta_{1}}\right\|_{L^{p_{1}(\cdot)}\left(\mathbb{R}^{n}\right)}\left\|\chi_{B_{j}}\right\|_{L^{p_{1}^{\prime} \cdot(\cdot)}}\left\|\chi_{B_{k}}\right\|_{L^{p_{2}(\cdot)}}\right]^{\left(q_{2}^{1}\right)_{k}} \\
& \leq C 2^{-k_{0} \lambda_{2}} \sum_{k=0}^{k_{0}}\left[2^{k \alpha(\cdot)} \sum_{j=0}^{k-2} 2^{-k(n-(\mu+\beta))} 2^{(k-j) \frac{n}{r}}\left\|\frac{\left|f_{j}\right|}{\eta_{1}}\right\|_{L^{p_{1}(\cdot)}\left(\mathbb{R}^{n}\right)}\left\|\chi_{B_{j}}\right\|_{L^{p_{1}^{\prime}(\cdot)}} 2^{-k(\mu+\beta)}\left\|\chi_{B_{k}}\right\|_{L^{p_{1}(\cdot)}}\right]^{\left(q_{2}^{1}\right)_{k}} \\
& \leq C 2^{-k_{0} \lambda_{2}} \sum_{k=-\infty}^{\infty}\left[2^{k \alpha(\cdot)} \sum_{j=0}^{k-2} 2^{(k-j) \frac{n}{r}}\left\|\frac{\left|f_{j}\right|}{\eta_{1}}\right\|_{L^{p_{1}(\cdot)}\left(\mathbb{R}^{n}\right)} \frac{\left\|\chi_{B_{j}}\right\|_{L^{p_{1}^{\prime}(\cdot)}}}{\left\|\chi_{B_{k}}\right\|_{L^{p_{1}^{\prime}(\cdot)}}}\right]^{\left(q_{2}^{1}\right)_{k}} \\
& \leq C 2^{-k_{0} \lambda_{2}} \sum_{k=0}^{\infty}\left[2^{k \alpha(\cdot)} \sum_{j=0}^{k-2} 2^{(j-k)\left(n \delta_{1}-\frac{n}{r}\right)}\left\|\frac{\left|f_{j}\right|}{\eta_{1}}\right\|_{L^{p_{1}(\cdot)}\left(\mathbb{R}^{n}\right)}\right]^{\left(q_{2}^{1}\right)_{k}} \\
& \left.\leq C 2^{-k_{0} \lambda_{2}} \sum_{k=0}^{\infty}\left[\sum_{j=0}^{k-2} 2^{(j-k)\left(n \delta_{1}-\frac{n}{r}-\alpha_{+}\right.}\right)\left\|\frac{2^{j \alpha_{+}}\left|f \chi_{k}\right|}{\eta_{1}}\right\|_{L^{p_{1}(\cdot)}\left(\mathbb{R}^{n}\right)}\right]^{\left(q_{2}^{1}\right)_{k}}
\end{aligned}
$$




$$
\left.\leq C 2^{-k_{0} \lambda_{2}} \sum_{k=0}^{\infty}\left[\sum_{j=0}^{k-2} 2^{(j-k)\left(n \delta_{1}-\frac{n}{r}-\alpha_{+}\right.}\right)\left\|\left(\frac{2^{j \alpha_{+}}\left|f \chi_{k}\right|}{\eta_{1}}\right)^{q_{1}(\cdot)}\right\|_{L^{\frac{q_{1}}{q_{1}(\cdot)}}\left(\mathbb{R}^{n}\right)}^{\frac{1}{\left(q_{1}^{1}\right) j}}\right]^{\left(q_{2}^{1}\right)_{k}}
$$

Notice that $f \in M K_{q_{1}(\cdot), p_{1}(\cdot)}^{\alpha_{+}, \lambda_{1}}\left(\mathbb{R}^{n}\right),\left(\lambda_{2}\right) /\left(q_{2}\right)_{+}=\left(\lambda_{1}\right) /\left(q_{1}\right)_{-}$and $\alpha_{+}<n \delta_{1}+\frac{n}{r}+\left(\lambda_{1}\right) /\left(q_{1}\right)_{-}$. In the same way as we estimated $\eta_{11}$ in Theorem 1 , we obtain that

$$
\begin{aligned}
& 2^{-k_{0} \lambda_{2}} \sum_{k=0}^{k_{0}}\left\|\left(\frac{2^{k \alpha(\cdot)}\left|\sum_{j=0}^{k-2} T_{\Omega, \mu}^{A}\left(f_{j}\right) \chi_{k}\right|}{\eta_{1} \sum_{|\gamma|=m}\left\|D^{\gamma} A\right\|_{\lambda_{\beta}}}\right)^{q_{2}(\cdot)}\right\|_{L^{\frac{p_{2}(\cdot)}{q_{2}(\cdot)}}} \\
& \leq C \sum_{k=0}^{\infty} 2^{\left(k-k_{0}\right) \lambda_{2}}\left[\sum_{j=0}^{k-2} 2^{(j-k)\left(\left(\lambda_{1}\right) /\left(q_{1}\right)_{-}+n \delta_{1}-\frac{n}{r}-\alpha_{+}\right)} \times\left(2^{-j \lambda} \sum_{n=0}^{j}\left\|\left(\frac{2^{n \alpha_{+}}\left|f \chi_{n}\right|}{\eta_{1}}\right)^{q_{1}(\cdot)}\right\|_{L^{\frac{p_{1}(\cdot)}{q_{1}(\cdot)}}\left(\mathbb{R}^{n}\right)}\right)^{\frac{1}{\left(q_{1}^{1}\right) j}}\right]^{\left(q_{2}^{1}\right)_{k}} \\
& \leq C
\end{aligned}
$$

where

$$
\left(q_{1}^{1}\right)_{j}=\left\{\begin{array}{l|l}
\left(q_{1}\right)_{-}, & \| \frac{2^{j \alpha_{+}}\left|f_{\chi_{j}}\right|}{\eta_{1}} \\
\left(q_{1}\right)_{+}, & \left\|\frac{2^{j \alpha_{+}}\left|f_{\chi_{j}}\right|}{\eta_{1}}\right\|_{L^{p_{1}(\cdot)}} \leq 1, \\
L^{p_{1}(\cdot)}>1 .
\end{array}\right.
$$

This implies that

$$
\eta_{21} \leq C \eta_{1} \sum_{|\gamma|=m}\left\|D^{\gamma} A\right\|_{\lambda_{\beta}}=\sum_{|\gamma|=m}\left\|D^{\gamma} A\right\|_{\lambda_{\beta}}\|f\|_{M K_{q_{1}(\cdot), p_{1}(\cdot)}^{\alpha(\cdot), \lambda_{1}}\left(\mathbb{R}^{n}\right)}
$$

Finally, we consider $\eta_{23}$. Let $x \in C_{k}, j \geq k+2$, then $|x-y| \sim|y|$, we have

$$
\left|T_{\Omega, \mu}^{A} f_{j}(x)\right| \leq \int_{C_{j}} \frac{|\Omega(x-y)|\left|f_{j}(y)\right|}{|x-y|^{n-\mu+m}}\left|R_{m+1}(A ; x, y)\right| \mathrm{d} y .
$$

Applying Lemma 10 and Hölder's inequality, then we have

$$
\begin{aligned}
\left|T_{\Omega, \mu}^{A} f_{j}(x)\right| & \leq C \sum_{|\gamma|=m}\left\|D^{\gamma} A\right\|_{\lambda_{\beta}} \int_{C_{j}} \frac{|\Omega(x-y)|\left|f_{j}(y)\right|}{|x-y|^{n-(\mu+\beta)}} \mathrm{d} y \\
& \leq C \sum_{|\gamma|=m}\left\|D^{\gamma} A\right\|_{\lambda_{\beta}} 2^{-j(n-(\mu+\beta))}\left\|f_{j}\right\|_{L^{p_{1}(\cdot)}}\left\|\Omega(x-y) \chi_{j}\right\|_{L^{p^{\prime}(\cdot)}} \\
& \leq C \sum_{|\gamma|=m}\left\|D^{\gamma} A\right\|_{\lambda_{\beta}} 2^{-j(n-(\mu+\beta))}\left\|f_{j}\right\|_{L^{p_{1}(\cdot)}}\left\|\chi_{B_{j}}\right\|_{L^{p_{1}^{\prime}(\cdot)}} .
\end{aligned}
$$

From this and applying Lemma 4 and Lemma 5, we conclude that

$$
\begin{aligned}
& 2^{-k_{0} \lambda_{2}} \sum_{k=0}^{k_{0}}\left\|\left(\frac{2^{k \alpha(\cdot)}\left|\sum_{j=k+2}^{\infty} T_{\Omega, \mu}^{A}\left(f_{j}\right) \chi_{k}\right|}{\eta_{1} \sum_{|\gamma|=m}\left\|D^{\gamma} A\right\|_{\lambda_{\beta}}}\right)^{q_{2}(\cdot)}\right\|_{L_{p_{2}(\cdot)}^{q_{2}(\cdot)}} \leq 2^{-k_{0} \lambda_{2}} \sum_{k=0}^{k_{0}}\left\|\frac{2^{k \alpha(\cdot)}\left|\sum_{j=k+2}^{\infty} T_{\Omega, \mu}^{A}\left(f_{j}\right) \chi_{k}\right|}{\eta_{1} \sum_{|\gamma|=m}\left\|D^{\gamma} A\right\|_{\lambda_{\beta}}}\right\|_{L^{p_{2}(\cdot)}}^{\left(q_{2}^{2}\right)_{k}} \\
& \leq C 2^{-k_{0} \lambda_{2}} \sum_{k=0}^{k_{0}}\left[2^{k \alpha(\cdot)} \sum_{j=k+2}^{\infty} 2^{2^{-j(n-(\mu+\beta))}}\left\|\frac{\left|f_{j}\right|}{\eta_{1}}\right\|_{L^{p_{1}(\cdot)\left(\mathbb{R}^{n}\right)}}\left\|\chi_{B_{j}}\right\|_{L^{p_{1}^{\prime}(\cdot)}}\left\|\chi_{B_{k}}\right\|_{L^{p_{2}(\cdot)}}\right]^{\left(q_{2}^{2}\right)_{k}} \\
& \leq C 2^{-k_{0} \lambda_{2}} \sum_{k=0}^{k_{0}}\left[2^{k \alpha(\cdot)} \sum_{j=k+2}^{\infty} 2^{2^{-j(n-(\mu+\beta))}}\left\|\frac{\left|f_{j}\right|}{\eta_{1}}\right\|_{L^{p_{1}(\cdot)\left(\mathbb{R}^{n}\right)}}\left\|\chi_{B_{j}}\right\|_{L^{p_{1}^{\prime}(\cdot)}}\left\|\chi_{B_{k}}\right\|_{L^{p_{2}(\cdot)}}\right]^{\left(q_{2}^{2}\right)_{k}} \\
& \leq C 2^{-k_{0} \lambda_{2}} \sum_{k=0}^{k_{0}}\left[2^{k \alpha(\cdot)} \sum_{j=k+2}^{\infty} 2^{2^{-j(n-(\mu+\beta))}}\left\|\frac{\left|f_{j}\right|}{\eta_{1}}\right\|_{L^{p_{1}(\cdot)\left(\mathbb{R}^{n}\right)}}\left\|\chi_{B_{j}}\right\|_{L^{p_{1}^{\prime}(\cdot)}} 2^{-k(\mu+\beta)}\left\|\chi_{B_{k}}\right\|_{L^{p_{1}(\cdot)}}\right]^{\left(q_{2}^{2}\right)_{k}}
\end{aligned}
$$




$$
\begin{aligned}
& \leq C 2^{-k_{0} \lambda_{2}} \sum_{k=0}^{k_{0}}\left[2^{k \alpha(\cdot)} \sum_{j=k+2}^{\infty} 2^{(j-k)(\mu+\beta)}\left\|\frac{\left|f_{j}\right|}{\eta_{1}}\right\|_{L^{p_{1}(\cdot)}\left(\mathbb{R}^{n}\right)} 2^{-j n}\left\|\chi_{B_{j}}\right\|_{L^{p_{1}^{\prime}(\cdot)}}\left\|\chi_{B_{k}}\right\|_{L^{p_{1}(\cdot)}}\right]^{\left(q_{2}^{2}\right)_{k}} \\
& \leq C 2^{-k_{0} \lambda_{2}} \sum_{k=0}^{k_{0}}\left[2^{k \alpha(\cdot)} \sum_{j=k+2}^{\infty} 2^{(j-k)(\mu+\beta)}\left\|\frac{\left|f_{j}\right|}{\eta_{1}}\right\|_{L^{p_{1}(\cdot)}\left(\mathbb{R}^{n}\right)} \frac{\left\|\chi_{B_{k}}\right\|_{L^{p_{1}(\cdot)}}}{\left\|\chi_{B_{j}}\right\|_{L^{p_{1}(\cdot)}}}\right]^{\left(q_{2}^{2}\right)_{k}} \\
& \leq C 2^{-k_{0} \lambda_{2}} \sum_{k=0}^{k_{0}}\left[2^{k \alpha(\cdot)} \sum_{j=k+2}^{\infty} 2^{(k-j)\left(n \delta_{2}-(\mu+\beta)\right)}\left\|\frac{\left|f_{j}\right|}{\eta_{1}}\right\|_{L^{p_{1}(\cdot)}\left(\mathbb{R}^{n}\right)}\right]^{\left(q_{2}^{2}\right)_{k}} \text {, }
\end{aligned}
$$

where

$$
\left(q_{2}^{2}\right)_{k}=\left\{\begin{array}{l|l}
\left(q_{2}\right)_{-}, & \left\|\left(\frac{2^{k \alpha(\cdot)}\left|\sum_{j=0}^{k-2} T_{\Omega, \mu}^{A}\left(f_{j}\right) \chi_{k}\right|}{\eta_{1} \sum_{|\gamma|=m}\left\|D^{\gamma} A\right\|_{\lambda_{\beta}}}\right)^{q_{2}(\cdot)}\right\|_{L^{p_{2}(\cdot)}} \leq 1, \\
\left(q_{2}\right)_{+}, & \left\|\left(\frac{2^{k \alpha(\cdot)}\left|\sum_{j=0}^{k-2} T_{\Omega, \mu}^{A}\left(f_{j}\right) \chi_{k}\right|}{\eta_{1} \sum_{|\gamma|=m}\left\|D^{\gamma} A\right\|_{\Lambda_{\beta}}}\right)^{q_{2}(\cdot)}\right\|_{L^{p_{2}(\cdot)}}>1 .
\end{array}\right.
$$

Notice that $f \in M K_{q_{1}(\cdot), p_{1}(\cdot)}^{\alpha_{+}, \lambda_{1}}\left(\mathbb{R}^{n}\right),\left(\lambda_{2}\right) /\left(q_{2}\right)_{+}=\left(\lambda_{1}\right) /\left(q_{1}\right)_{-}$and $\alpha_{+}>(\mu+\beta)-n \delta_{2}+\left(\lambda_{1}\right) /\left(q_{1}\right)_{-}$, by the same argument as that of $\eta_{13}$, we have

$$
\begin{aligned}
& 2^{-k_{0} \lambda_{2}} \sum_{k=0}^{k_{0}}\left\|\left(\frac{2^{k \alpha(\cdot)}\left|\sum_{j=k+2}^{\infty} T_{\Omega, \mu}^{A}\left(f_{j}\right) \chi_{k}\right|}{\eta_{1} \sum_{|\gamma|=m}\left\|D^{\gamma} A\right\|_{\lambda_{\beta}}}\right)^{q_{2}(\cdot)}\right\|_{L^{\frac{p_{2}(\cdot)}{q_{2}(\cdot)}}} \\
& \leq C 2^{-k_{0} \lambda_{2}} \sum_{k=0}^{\infty}\left[\sum_{j=k+2}^{\infty} 2^{(k-j)\left(n \delta_{2}-(\mu+\beta)+\alpha_{+}\right)}\left\|\left(\frac{2^{j \alpha_{+}}\left|f \chi_{k}\right|}{\eta_{1}}\right)^{q_{1}(\cdot)}\right\|_{L^{\frac{1}{q_{1}^{2}(\cdot)}}\left(\mathbb{R}^{n}\right)}^{\left(q_{1}^{2}\right)}\right]^{\left(q_{2}^{2}\right)_{k}} \\
& \leq C \sum_{k=0}^{\infty} 2^{\left(k-k_{0}\right) \lambda_{2}}\left[\sum_{j=k+2}^{\infty} 2^{(k-j)\left(n \delta_{2}-(\mu+\beta)-\left(\lambda_{1}\right) /\left(q_{1}\right)_{-}+\alpha_{+}\right)}\right. \\
& \left.\times\left(2^{-j \lambda} \sum_{n=0}^{j}\left\|\left(\frac{2^{n \alpha_{+}}\left|f \chi_{n}\right|}{\eta_{1}}\right)^{q_{1}(\cdot)}\right\|_{L^{\frac{p_{1}(\cdot)}{q_{1}(\cdot)}}\left(\mathbb{R}^{n}\right)}\right)^{\frac{1}{\left(q_{1}^{2}\right) j}}\right]^{\left(q_{2}^{2}\right)_{k}} \\
& \leq C,
\end{aligned}
$$

where

$$
\left(q_{1}^{2}\right)_{j}=\left\{\begin{array}{l|l}
\left(q_{1}\right)_{-}, & \left\|\frac{2^{j \alpha+}\left|f_{\chi_{j}}\right|}{\eta_{1}}\right\|_{L^{p_{1}(\cdot)}} \leq 1, \\
\left(q_{1}\right)_{+}, & \left\|\frac{2^{j \alpha} \mid f_{\chi_{j}}}{\eta_{1}}\right\|_{L^{p_{1}(\cdot)}}>1 .
\end{array}\right.
$$

This implies that

$$
\eta_{23} \leq C \eta_{1} \sum_{|\gamma|=m}\left\|D^{\gamma} A\right\|_{\lambda_{\beta}}=\sum_{|\gamma|=m}\left\|D^{\gamma} A\right\|_{\lambda_{\beta}}\|f\|_{M K_{q_{1}(\cdot), p_{1}(\cdot)}^{\alpha(\cdot), \lambda_{1}}\left(\mathbb{R}^{n}\right)} .
$$

Then, the proof of Theorem 2 is finished.

Acknowledgments: The author wishes to express profound gratitude to the reviewers for their useful comments on the manuscript.

Author Contributions: All authors contributed equally to the writing of this paper. All authors read and approved the final manuscript.

Conflicts of Interest: The authors declare no conflict of interest.

\section{References}


[1] Coifman, R. R., \& Meyer, Y. (1975). On commutators of singular integrals and bilinear singular integrals. Transactions of the American Mathematical Society, 212, 315-331.

[2] Wu, Q., \& Yang, D. (2002). On fractional multilinear singular integrals. Mathematische Nachrichten, 239(1), $215-235$.

[3] Tao, X., \& Wu, Y. (2012). BMO estimate for multilinear fractional integrals. Analysis in Theory and Applications, 28, 224-231.

[4] Ding, Y., Lu, S. Z., \& Yabuta, K. (2006). Multilinear singular and fractional integrals. Acta Mathematica Sinica, 22(2), 347-356.

[5] Li, Q., \& Tao, S. (2013). Boundedness of multilinear commutators with rough kernels on Morrey-Herz spaces. Analysis in Theory and Applications 39, 289-307.

[6] Kováčik, O., \& Rákosník, J. (1991). On Spaces $L^{p(x)}$ and $W^{k, p(x)}$. Czechoslovak Mathematical Journal, 41 ,592-618.

[7] Cruz-Uribe, D. V., \& Fiorenza, A. (2013). Variable Lebesgue spaces: Foundations and harmonic analysis. Springer Nature Switzerland.

[8] Wu, H., \& Lan, J. (2012). The boundedness for a class of rough fractional integral operators on variable exponent Lebesgue spaces. Analysis in Theory and Applications, 28(3), 286-293.

[9] Diening, L., Harjulehto, P., Hästö, P., \& Ruzicka, M. (2011). Lebesgue and Sobolev spaces with variable exponents. Springer Nature Switzerland.

[10] Wang, H., Wang, J., \& Fu, Z. (2017). Morrey meets Herz with variable exponent and applications to commutators of homogeneous fractional integrals with rough kernels. Journal of Function Spaces, 2017, Article ID 1908794, 11 pages.

[11] Izuki, M. (2010). Boundedness of sublinear operators on Herz spaces with variable exponent and application to wavelet characterization. Analysis Mathematica, 36(1), 33-50.

[12] Abdalmonem, A., Abdalrhman, A., \& Tao, S. (2018). Boundedness of fractional integral marcinkiewicz integral with variable kernel on variable Morrey-Herz Spaces. Open Journal of Mathematical Sciences, 2(1),93-114.

[13] Lijuan, W., \& Tao, S. (2016). Parameterized Littlewood-Paley operators and their commutators on Morrey- Herz spaces with variable exponents. Turkish Journal of Mathematics, 40(1), 122-145.

[14] Wu, H. L., \& Lan, J. C. (2013). Lipschitz estimates for fractional multilinear singular integral on variable exponent Lebesgue spaces. Abstract and Applied Analysis, 1013, Article ID 632384, 6 pages.

[15] Xu, J. S. (2007). The boundedness of multilinear commutators of singular integrals on Lebesgue spaces with variable exponent. Czechoslovak Mathematical Journal, 57(1), 13-27.

[16] Lu, Y., \& Zhu, Y. (2014). Boundedness of multilinear calderón-Zygmund singular operators on Morrey-Herz spaces with variable exponents. Acta Mathematica Sinica, 30,1180-1194.

[17] Min, W., Meng, Q., \& Sheng, S. (2015). Multilinear fractional integral operators on Morrey spaces with variable exponent on bounded domain. Communications in Mathematical Research, 31, 253-260.

[18] Wang, L., \& Tao, S. (2015). Parameterized Littlewood-Paley operators and their commutators on Lebegue spaces with variable exponent. Analysis in Theory and Applications, 31, 13-24.

[19] Jian, T. A. N., \& Liu, Z. G. (2015). Some Boundedness of Homogeneous Fractional Integrals onVariable Exponent Function Spaces. ACTA Mathematics Science (Chinese Series), 58, 310-320.

[20] Izuki, M. (2010). Boundedness of commutators on Herz spaces with variable exponent. Rendiconti del Circolo Matematico di Palermo, 59(2), 199-213.

[21] Cohen, J., \& Gosselin, J. (1986). A BMO estimate for multilinear singular integrals. Illinois Journal of Mathematics, 30(3), 445-464.

[22] Ho, K. P. (2013). The fractional integral operators on Morrey spaces with variable exponent on unbounded domains. Mathematical Inequalities E Applications, 16, 363-373.

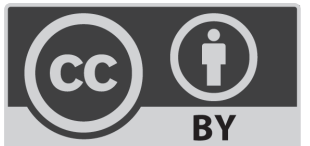

(C) 2019 by the authors; licensee PSRP, Lahore, Pakistan. This article is an open access article distributed under the terms and conditions of the Creative Commons Attribution (CC-BY) license (http://creativecommons.org/licenses/by/4.0/). 